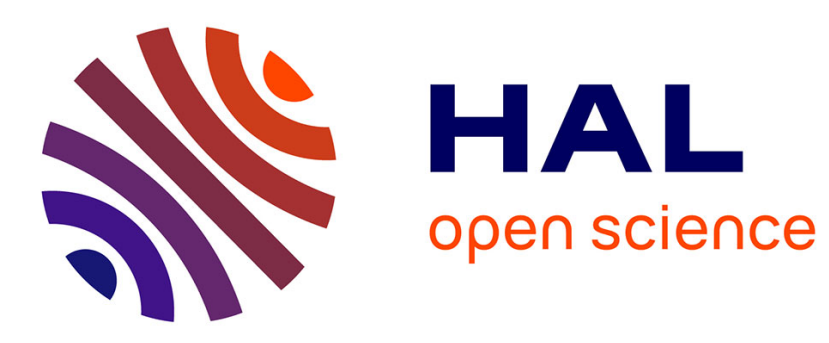

\title{
Frontal Instabilities at Density-Shear Interfaces in Rotating Two-Layer Stratified Fluids
}

\author{
Hélène Scolan, Roberto Verzicco, Jan-Bert Flór
}

\section{To cite this version:}

Hélène Scolan, Roberto Verzicco, Jan-Bert Flór. Frontal Instabilities at Density-Shear Interfaces in Rotating Two-Layer Stratified Fluids. Thomas von Larcher; Paul D. Williams. Modeling Atmospheric and Oceanic Flows: Insights from Laboratory Experiments and Numerical Simulations, American Geophysical Union, 2014, Geophysical Monograph Series, 9781118855935. 10.1002/9781118856024.ch11 . hal-01875887

\section{HAL Id: hal-01875887 \\ https://hal.science/hal-01875887}

Submitted on 19 Feb 2020

HAL is a multi-disciplinary open access archive for the deposit and dissemination of scientific research documents, whether they are published or not. The documents may come from teaching and research institutions in France or abroad, or from public or private research centers.
L'archive ouverte pluridisciplinaire HAL, est destinée au dépôt et à la diffusion de documents scientifiques de niveau recherche, publiés ou non, émanant des établissements d'enseignement et de recherche français ou étrangers, des laboratoires publics ou privés. 


\title{
Frontal Instabilities at Density-Shear Interfaces in Rotating Two-Layer Stratified Fluids
}

\author{
Hélène Scolan ${ }^{1}$, Roberto Verzicco ${ }^{2}$, and Jan-Bert Flór ${ }^{3}$
}

\subsection{INTRODUCTION}

Fronts in Earth oceans and atmosphere separate different temperature fluids or masses of air and therefore play a major role for the transport of heat and chemical or biological tracers in large-scale geophysical flows. Their dynamics and instability are crucial for weather forecasting, whereas their variability is a key element for understanding climate. First experiments that showed the occurrence of baroclinic modes were conducted in annular rotating tanks of which the exterior (or inner) cylinder was heated (cooled) [Hide, 1953, 1958; Fultz et al., 1959; Fowlis and Hide, 1965].

Baroclinic instability arises at a density field that is inclined with respect to the horizontal. As mentioned, an inclined density gradient may be created in a rotating fluid by differential heating. In the quasi-geostrophic approximation, these flows are in thermal wind balance, i.e, the tilting of the vorticity due to shear in the fluid equals the baroclinic production of vorticity,

$$
f \frac{\partial \vec{u}}{\partial z}=-\frac{g}{\rho_{o}} \vec{z} \times \vec{\nabla}_{H} \rho,
$$

where $u$ is the horizontal velocity, $g$ the gravitational constant, and $\vec{\nabla}_{H} \rho$ the horizontal density gradient. Another manner to create a baroclinic front in thermal wind balance is achieved by applying a vertical shear across the density field. Such a shear can be obtained by using a rotating disk at the fluid surface [Hart, 1972]. For this type of flow the quasi-geostrophic two-layer model of

\footnotetext{
${ }^{1}$ Atmospheric, Oceanic \& Planetary Physics, University of Oxford, Oxford, United Kingdom.

${ }^{2}$ Department of Mechanical Engineering, Università di Roma Tor Vergata, Rome, Italy.

${ }^{3}$ Laboratoire des Ecoulements Géophysiques et Industriels (LEGI), Grenoble, France.
}

Phillips [1954] and Pedlosky [1964, 1970] [see Hart, 1972] predicts well the observed wavelengths of the baroclinic instability. These flows depend on stratification, rotation, and the flow aspect ratio as expressed by the Burger number. This number indicates the baroclinicity of the flow. Strongly baroclinic flows have a small Burger number corresponding to a strongly inclined density field. This number serves as a control parameter for baroclinic instability (see Section 11.2.1). Various aspects of baroclinic instability have been considered. For small Burger numbers, periodic variations occur in the amplitude of the dominant baroclinic mode of the system, a nonlinear effect known as amplitude vacillation [Hart, 1980, 1985; King, 1979]. In considering the same forcing at the fluid surface for an annular flow, also Bradford et al. [1981], Appleby [1982], Lovegrove et al. [2000], and Williams et al. [2003, 2004a, 2004b, 2005] showed cases of amplitude vacillation, whereas for even smaller Burger numbers the flow is known to transit to chaos and turbulence [Hide, 2011; Read et al., 1992; Früh and Read, 1997; Eccles et al., 2009].

Other examples of fronts where the baroclinic instability is observed to develop are density intrusions in a rotating fluid, such as gravity currents, coastal flows, and vortex lenses created by the release of a fluid of a different density in a homogeneous (or stratified) rotating ambient [Chia et al., 1982; Bouruet-Aubertot and Linden, 2002]. These systems generally consist of two layers, and the Phillips model for baroclinic instability shows good agreement with the observed instability wavelengths [Griffiths and Linden, 1981; Cenedese and Linden, 2002]. Interactions of the front with the Ekman boundary layer do not seem to influence the instability wavelength of these close to laminar fronts in small-scale laboratory experiments. Also the complex dynamics of critical layers at the interface do not significantly modify observations of the 
Table 11.1. Dimensions and parameter regimes of experiments on the instability of baroclinic fronts and the parameter ranges considered.

\begin{tabular}{|c|c|c|c|c|c|c|c|}
\hline Reference & $\Gamma$ & $\gamma$ & $L(\mathrm{~cm})$ & $\mathrm{Bu}$ & Ro & $E_{k}$ & $d$ \\
\hline $\begin{array}{l}\text { Hart [1972, 1973, 1976, } \\
1979,1980,1981,1985]\end{array}$ & $0.15,0.039$ & 0.36 or 0.67 & 12.5 & $0.02-0.1$ & $0.02-0.25$ & $\begin{array}{l}2.5 \times 10^{-7}, 3 \times 10^{-3} \\
3 \times 10^{-5}\end{array}$ & $0.1-0.7$ \\
\hline King [1979] & $0.01-0.02$ & 2.19 & 5.7 & $0.04-0.125$ & $0.035-0.1$ & $1 \times 10^{-7}-1 \times 10^{-5}$ & $0.1-0.7$ \\
\hline Carrigan [1978] & 0.027 & 0.67 & 7.62 & $0.0033-0.033$ & $0.017-0.5$ & $1 \times 10^{-3}-1 \times 10^{-2}$ & - \\
\hline Lovegrove et al. [1999, 2000] & 0.006 & 2 & 6.25 & $0.06-0.25$ & $0.1-10$ & $1 \times 10^{-6}-1 \times 10^{-4}$ & $0.004-0.03$ \\
\hline $\begin{array}{l}\text { Williams et al. [2003, 2004a, } \\
\text { 2004b, 2005, 2008, 2010] }\end{array}$ & 0.006 & 2 & 6.25 & $0.03-333$ & $0.1-100$ & $1 \times 10^{-6}-3 \times 10^{-3}$ & $0.0003-0.03$ \\
\hline Flór et al. [2011] & 0.006 & 0.18 & 75 & $0.02-5$ & $0.06-1.5$ & $7 \times 10^{-5}-7 \times 10^{-4}$ & $0.01-0.1$ \\
\hline
\end{tabular}

Note: Flór et al. [2011] considered miscible salt-stratified fluids.

baroclinic instability. In this chapter, we focus on fronts that do not intersect with the top or bottom boundary.

We consider ageostrophic flows and discuss the recently investigated Rossby-Kelvin (RK) instability first discovered by Sakai [1989] and aspects of the frontal instability that are related to the thickness of the interface. Sakai [1989] considered ageostrophic modeling of baroclinic instability and, following Hayashi and Young [1987], investigated the resonance of different type of waves, such as Kelvin, Rossby, and Poincaré waves. The baroclinic instability was interpreted as a resonance (a coupling of the phase speeds) between two Rossby waves, one in the upper and one in the lower layer. The RK instability is a consequence of the resonance between a Rossby wave in one layer and Kelvin or Poincaré wave in the other layer. Though gravity waves move faster than Rossby waves, resonance occurs when the two wave frequencies match due to the Doppler shift. Recently, this approach has been continued for rectangular and annular geometries respectively by Gula et al. [2009a, 2009b], Gula and Zeitlin [2010], and Gula et al. [2010] showing especially significant growth rates for the resonance of a Rossby wave in the upper layer and a coastal Kelvin wave in the lower layer. Sakai [1989] used the term Rossby-Kelvin for all vorticitygravity wave resonances, but here we will reduce the term "Rossby-Kelvin instability" to this particular resonance. These results were compared to experimental results by Flór et al. [2011].

The choice of a fluid interface between two miscible fluid layers is in between the limits of a very thin immiscible fluid interface [Williams et al., 2005] and, at the other end, a linear density stratification. For two-layer fluids, it has been shown in numerical simulations [Gula et al., 2009a] that the RK instability occurs only for thin interfaces. For interfaces with a thickness beyond a certain threshold, i.e. $\delta_{\rho} /(2 H)>0.16$, where $2 H$ is the fluid depth and $\delta_{\rho}$ the interface thickness (see Section 11.3.2), the growth rate of ageostrophic modes was found to reduce to zero. Though we are not aware of simulations on the RK instability in linearly stratified fluids, in considering a very thick interface as an approximation of a linear stratification, one may expect also a zero growth rate there. In general, relatively thin density interfaces allow for a richer variety of shear instabilities, including KelvinHelmholtz and Hölmböe instability (see Section 11.3.3).

Small-scale waves at the immiscible fluid interface in the baroclinic unstable regime [Lovegrove et al., 2000, Williams et al., 2003, 2004a, 2004b, 2005] were interpreted as the spontaneous emission of inertia-gravity waves. In these experiments the optical activity of the two immiscible fluids gave access to very accurate measurements of interfacial perturbations. A constraint was that the reduced gravity was fixed by the limited type of optical fluids that is available (see Table 11.1). In a larger setup and filled with a salt-stratified two-layer fluid [Flór et al., 2011], interfacial waves due to surface tension effects were eliminated, and, as mentioned, access to a larger range of scales of motion was achieved. Hölmböe instability was in part considered responsible for similar type of small waves, a mechanism that could also be efficient in the immiscible fluid experiments mentioned above [Flór et al., 2011].

In this chapter, we report numerical results for a twolayer stratification with a smooth interface that have earlier been presented by Scolan [2011], and experimental results by Flór et al. [2011]. We discuss these interfacial waves next to the different instabilities observed in the parameter space set by Burger number, Rossby, and Ekman or the dissipation number, defined in the next section. In doing so, we consider experimental and numerical results of the setup depicted in Figure 11.1.

The governing equations and pertinent nondimensional numbers of this experimental system are presented in Section 11.2.1, followed by a brief description of the numerical approach in Section 11.2.2. The instability regime diagrams and the baroclinic instability including the recently reported observations of the RK instability are presented in Section 11.3.1. In Section 11.3.2 the secondary vertical circulation is investigated numerically. 


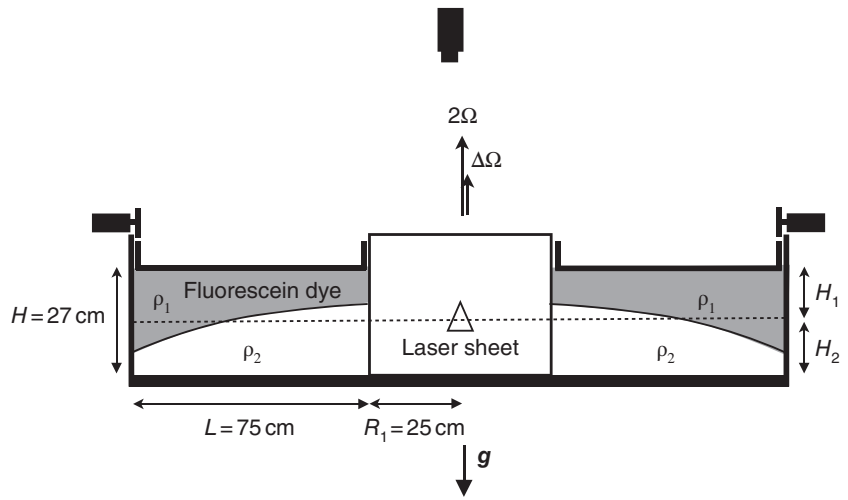

Figure 11.1. Sketch of experimental setup, with $\rho_{1}$ and $\rho_{2}$ the upper and lower layer fluid densities of depth $H$; the annular disk at the surface is driven by three motors with rotation $\Delta \Omega$.

We show the presence of interfacial Ekman layers and the influence of the Schmidt number. These properties are relevant for the small-scale instabilities discussed in Section 11.3.3. When the internal fluid interface is not discrete but continuous as in a salt-stratified two-layer fluid, questions about instability such as Kelvin-Helmholtz and Hölmböe instability, internal Ekman layers, as well as the effect of stratification on the RK instability come into play (Section 11.3.3). In the conclusions (Section 11.4) we discuss the different results in a geophysical context in considering realistic Burger and Rossby numbers in the atmosphere and oceans and a range of different experiments on baroclinic (Figure 11.2) instabilities, and small-scale instabilities.

\subsection{EQUATIONS AND SCALES FOR LABORATORY EXPERIMENTS}

The dynamics for a two-layer salt-stratified and rotating fluid with viscous boundary effects is described by the Navier-Stokes equations with boundary conditions. They are composed of the continuity equation, the equation of motion (including Coriolis force and centrifugal forces), and the conservation of salt. In the Boussinesq approximation, the general equations for a salt-stratified fluid in rotation are

$$
\begin{aligned}
\nabla \cdot \vec{u}= & 0, \\
\frac{\partial \vec{u}}{\partial t}+(\vec{u} \cdot \nabla) \vec{u}= & -\frac{\nabla p}{\rho_{o}}-2 \vec{\Omega} \times \vec{u}-g \frac{\rho-\rho_{o}}{\rho_{o}} \hat{\mathbf{z}}+v \nabla^{2} \vec{u} \\
& +\frac{\rho-\rho_{o}}{\rho_{o}} \Omega^{2} r \hat{\mathbf{r}}, \\
\frac{\partial S}{\partial t}+\nabla \cdot(S \vec{u})= & \kappa \nabla^{2} S,
\end{aligned}
$$

with $\vec{u}$ and $p$, the velocity and pressure field, respectively, $\rho_{o}$ the mean density, i.e., $\rho_{o}=\left(\rho_{1}+\rho_{2}\right) / 2$, and the density field is assumed to vary as $\rho=\rho_{o}+\tilde{\rho}$ with the density perturbation $\tilde{\rho}$ assumed to be proportional to the salinity $S$, i.e. $\tilde{\rho}(\vec{r})=\alpha S(\vec{r})$. The diffusivity of this scalar quantity $S$ is represented by $\kappa$.

Following Hart [1972] the equations are nondimensionalized with length scale $L=R_{2}-R_{1}$, velocity scale $U=$ $\Delta \Omega L$, pressure $P=\rho_{1} U^{2}$, time scale $T=1 / \Delta \Omega$, and for the density (salt concentration) $\Delta \rho=\rho_{2}-\rho_{1}$. The experimental setup and notations are represented in Figure 11.1. The scaled equations then become

$$
\begin{aligned}
\frac{\partial \vec{u}}{\partial t}+(\vec{u} \cdot \nabla) \vec{u}= & -\nabla p-\frac{1}{R_{o}} \hat{\mathbf{k}} \times \vec{u}-\frac{B_{u}}{R_{o}^{2} H / L} S \hat{\mathbf{z}}+\frac{1}{R_{e}} \nabla^{2} \vec{u} \\
& +\frac{g^{\prime} / g}{4 R_{o}^{2}} S r \hat{\mathbf{r}}, \\
\frac{\partial S}{\partial t}+\nabla \cdot(S \vec{u})= & \frac{1}{P e} \nabla^{2} S
\end{aligned}
$$

with the nondimensional parameters defined as the Burger number (or Froude number $\mathrm{Fr}=1 / \mathrm{Bu}$ )

$$
\mathrm{Bu}=\frac{g^{\prime} H}{4 \Omega^{2} L^{2}},
$$

the Rossby number

$$
\text { Ro }=\frac{\Delta \Omega}{2 \Omega},
$$

and the Ekman number

$$
\mathrm{Ek}=\frac{v}{2 \Omega H^{2}},
$$

(a)

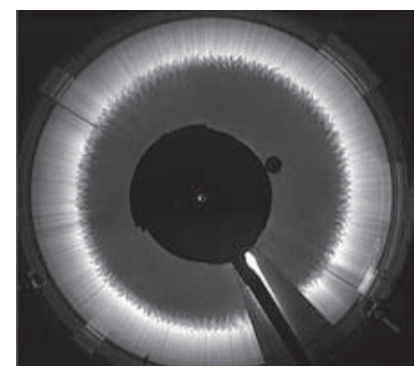

(c)

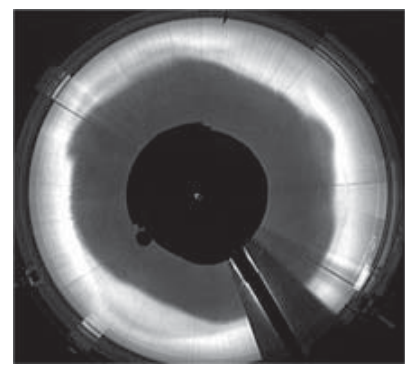

(b)

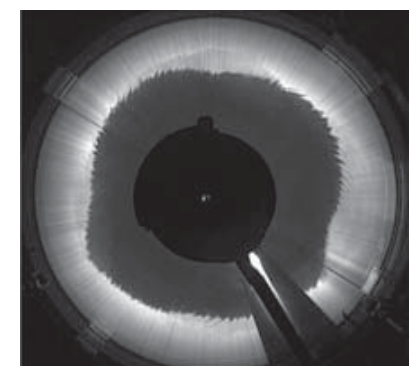

(d)

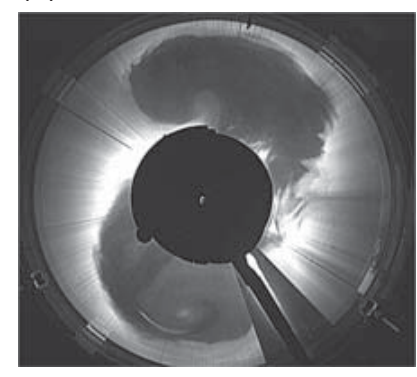

Figure 11.2. Typical observations of the instabilities reported in the diagram of Figure 11.3 with (a) the Hölmböe instability $(\mathrm{H})$, (b) and (c) respectively, mode 4 and mode 6 of the RK instability, and (d) the baroclinic instability (BI) from Flór et al. [2011]. 
where $H$ is the height for each layer, $g^{\prime}$ the reduced gravity $g^{\prime}=2 g\left(\rho_{2}-\rho_{1}\right) /\left(\rho_{2}+\rho_{1}\right), \Omega$ the background rotation and $\Delta \Omega$ the differential rotation of the disk. Often the dissipation number

$$
d=\frac{\sqrt{\nu \Omega}}{H \Delta \Omega}=\frac{\sqrt{E_{k}}}{\sqrt{2} \text { Ro }}
$$

is considered [Hart, 1972]. This number represents the dissipation of the spinning disk motion at the surface due to Ekman pumping. Alternatively, one can also consider the Reynolds number

$$
R_{e}=\frac{R_{o}}{E_{k}}\left(\frac{L}{H}\right)^{2}
$$

as dissipation parameter. For a given geometric configuration and stratification, the three other parameters are the ratio of accelerations $\Gamma=g^{\prime} / g$, the aspect ratio $\gamma=H / L$, and for the diffusivity of momentum compared to salt, the Schmidt number $\mathrm{Sc}=\nu / \kappa$. Instead of the Schmidt number, one can also consider the Peclet number $\mathrm{Pe}=\Delta \Omega L^{2} / \kappa=\mathrm{Re} \cdot \mathrm{Sc}$.

Given an experimental setup, the flow can be determined by three parameters, $d, \mathrm{Bu}$, and the Rossby number Ro. In experiments with immiscible fluids, $g^{\prime}$ is set by the available optically active fluids so that these experiments are associated with a Bu-Ro diagram (for a given range of rotation frequencies). A different reduced gravity $g^{\prime}$ would allow for the same Bu number but different Ro, Ek, or $d$ numbers. The complete regime diagram is therefore set by $\mathrm{Bu}, \mathrm{Ro}$, and $d$. When viscous effects are negligible, this reduces to a two-dimensional diagram spanned by Burger and Rossby number. In order to compare different experiments, in Flór et al. [2011], the parameters were chosen equal to those in the work of Williams et al. [2005]. Because of the miscible fluid interface and larger setup, we expect that threshold critical values may vary in this regime diagram. Here, the nondimensional parameters are the same as before, but for a cylindrical tank, the length scale $L$ is taken equal to the tank radius. Table 11.1 gives the experimental values of these systems so far tested in different experimental setups.

To examine the small-scale shear instabilities at the interface, we use the definition of Alexakis [2005] for the global Richardson number $J_{o}$, i.e. the gradient Richardson number $\mathrm{Ri}$ at the interface,

$$
J_{o}=\operatorname{Ri}(0)=J R=\frac{g^{\prime} \delta_{v}}{(\Delta U)^{2}} R,
$$

with the usual bulk Richardson number $J$, the total shear thickness $\delta_{v}$, and total density thickness $\delta_{\rho}$ of the interface and their ratio defined as $R=\delta_{v} / \delta_{\rho}$. In immiscible fluid layers, the total density thickness is given by twice the thickness of the Ekman layer.
From the general equations (11.1), the two-layer quasigeostrophic model was derived to study the baroclinic instability of a geostrophic flow analytically. This simplified model can be obtained from an asymptotic development of the equations in the limit of small Rossby numbers [Pedlosky, 1987]. At order zero, the flow simply verifies the geostrophic and hydrostatic balances. At order one, the development gives the quasi-geostrophic equations. This two-layer model gives major properties for the baroclinic instability and allowed predictions for the threshold Burger number for the baroclinic instability in Hart's experiments [Hart, 1972]. For layers of the same depth and same viscosity, the potential vorticity equations for the two-layer quasi-geostrophic flow including Ekman boundary layers [Hart, 1972; Pedlosky, 1970],

$$
\begin{aligned}
& \frac{d}{d t_{1}}\left[\nabla^{2} \psi_{1}+\frac{1}{\mathrm{Bu}}\left(\psi_{2}-\psi_{1}\right)\right]=-d\left(\frac{3}{2} \nabla^{2} \psi_{1}-\frac{1}{2} \nabla^{2} \psi_{2}-2\right), \\
& \frac{d}{d t_{2}}\left[\nabla^{2} \psi_{2}+\frac{1}{\mathrm{Bu}}\left(\psi_{1}-\psi_{2}\right)\right]=-d\left(\frac{3}{2} \nabla^{2} \psi_{2}-\frac{1}{2} \nabla^{2} \psi_{1}\right),
\end{aligned}
$$

with

$$
\frac{d}{d t_{i}}=\left[\frac{\partial}{\partial t}+J\left(\psi_{i},\right)\right],
$$

where $J$ is the Jacobian operator and $\psi_{i}$ the stream function in cylindrical coordinates for the layer $i$. The same dimensional parameters $\mathrm{Bu}$ and $d$ appear in the equations. The coupling between the layers occurs via the Burger number term and viscous effects in the form of Ekman pumping. Topographic effects are neglected since the bottom and disk are flat, and centrifugal effects on the shape of the interface are neglected [Hart, 1973]. Because of the quasi-geostrophic balance, inertia-gravity waves are filtered out, and the equations apply to length scales that are of the order of the deformation radius, for example, the scale of the Rossby waves.

By contrast, in the two-layer shallow-water model studied by Sakai [1989] and more recently by Gula and Zeitlin [2010] and Gula et al. [2010], gravity waves are not filtered out. Since this model contains no Rossby-number approximation or asymptotic development, it includes ageostrophic motions. It is an inviscid model and the effects due to Ekman pumping are neglected.

The quasi-geostrophic and the shallow-water model both rely on the shallow-water approximation, which assumes thin layers with a small aspect ratio and small vertical compared to horizontal gradients. The validity of this approximation in experimental configurations is achieved as long as $\left(H_{j} / L\right)^{2} \mathrm{Ro}_{j}$ in layer $j$ is small. For $H_{1}=H_{2}$, it corresponds to the product of the square of the aspect ratio, $\gamma^{2}$, with the Rossby number. This number gives the ratio between the acceleration of the vertical velocity and the vertical pressure gradient. For 
aspect ratio 0.18 in each layer and Rossby number between 0.1 and 1 , we have $\gamma^{2}$ Ro between 0.003 and 0.03 , which ensures that (a) the vertical acceleration can be neglected, (b) the hydrostatic balance is verified, and (c) the shallowwater approximation is valid. This condition is not always met in former experiments with larger aspect ratios (see Table 11.1).

Both models consider a jump in density with separated equations for each layer coupled via pressure and, if included, viscous effects. The dynamics of miscible interfaces are however not captured, and especially interactions between density and vorticity at the interface with shear instabilities are not included. Below we consider experiments and direct numerical simulations (DNSs) built on the full Boussinesq Navier-Stokes equations (11.2) in the annular configuration (see Section 11.2.2).

\subsubsection{Experimental Modeling of Fronts}

A baroclinic front in thermal wind balance is created in a two-layer rotating fluid with the shear across the interface driven by a rotating lid at the fluid surface. For the study of baroclinic instability, this forcing is preferable because the density difference is set from the beginning, and flow measurements of the velocity field and the density field are better accessible. The mixing of the miscible interface amounted to a very small percentage of the volume of the layers and therefore has a negligible influence on the buoyancy frequency at the interface. The flow dimensions of the experimental setup are indicated in Figure 11.1 and the parameter ranges are indicated in Table 11.1. In order to investigate the frontal evolution for the $\mathrm{Bu}$ number ranges indicated in Table 11.1, the disk rotation $\Delta \Omega$ was set constant for a very slowly increasing background rotation $\Omega(t)$ such that the values of the Ro, Ek, and Bu numbers changed correspondingly. This same method had initially been tested successfully by, e.g., Williams et al. [2005], and the comparison with the same experiment for fixed background rotation assured that Ekman pumping effects due to spin-up did not affect the flow dynamics and instability. The density difference across the interface, $\Delta \rho=\rho_{1}-\rho_{2}$, and reduced gravity, $g^{\prime}=g \Delta \rho / \bar{\rho}$ (with $g$ the gravity constant), were kept constant and close or equal to the values by Williams et al. [2005] (see Table 11.1).

To visualize the flow, the upper fluid layer was dyed with fluorescein dye and illuminated by a horizontal laser sheet at middepth such that the inclined interface of the front was visible in the top-view images. The wavelengths, phase speeds, and growths of the instabilities are obtained from spatiotemporal sequences of the top-view frontal evolution, also known as Hövmüller diagrams. In addition, PIV (Particle Image Velocimetry) measurements from tracer particles gave access to typical velocity fields in each layer. The resolution in the experiments, especially the dye visualizations, was of the order of $3 \mathrm{~mm}$ for a tank of $1 \mathrm{~m}$ radius and allowed to measure the presence of small-scale waves.

The lack of information about the flow characteristics such as the local density gradient and three-dimensional velocity field motivated us to obtain additional flow information from DNSs for a series of almost identical, or at least comparable, initial values as in the experiments.

\subsubsection{Numerical Approach}

The numerical simulations were performed using the DNS code of Verzicco et al. [1997]. The governing equations are the Boussinesq equations, including Coriolis and centrifugal forces in the annular configuration. Equations are written in cylindrical coordinates $\left(v_{r}, v_{\theta}, v_{z}\right)$ and discretized on a staggered mesh by central secondorder accurate finite difference approximation. Details of the numerical scheme are described by Verzicco and Orlandi [1996] and Verzicco and Camussi [1997]. The initial (nondimensional) density profiles are $\rho_{2}=1$ and $\rho_{1}=0$ to which a random value is added as a perturbation. At $t=0$, the velocity field $\left(v_{r}, v_{\theta}, v_{z}\right)$ is at rest. At the solid walls, the no-slip condition is used, except for the top boundary, $z=2 H$, where the rotating disk imposes an azimuthal velocity $v_{\theta}=\Delta \Omega$.

This code has been tested for different initial conditions. The resolution of the grid amounted in most 3D simulations $N_{\theta} \times N_{r} \times N_{z}=257 \times 97 \times 257$ or $97 \times 97 \times 97$. Tests with 2 times higher resolution grids as well as with 2 times lower resolution grids showed essentially the same results but with less detail.

In DNS, the full equations are solved, but care must be taken with the evolution at small scales. To assure stability, the grid size must be of the same order of magnitude as the Kolmogorov scale, i.e. the scale at which viscosity dissipates energy, $L_{K}=\mathrm{Re}^{-3 / 4} \times l$, with $l$ the integral scale and Re the Reynolds number. If the grid scale is larger than $L_{K}$, energy may accumulate artificially at this length scale without being dissipated, leading to a numerical instability. In the laboratory experiments, the Reynolds number based on the velocity of the rotated disk is of $O(50,000)$. Since this Reynolds number would require too long calculation times, a smaller Reynolds has been used that is based on a larger viscosity and a grid size that is adapted to the small-scale flow features of interest, as discussed below.

To reproduce the dynamics of the front in coherence with the laboratory experiments $(\mathrm{Sc}=700)$, the Schmidt number is necessarily large and requires a high resolution. For small Schmidt numbers, the density interface was found to diffuse too rapidly, i.e., before the front becomes unstable. For high Sc numbers, scalar diffusion is much weaker than viscous diffusion so that its typical length scale is also much smaller than the Kolmogorov scale. 
(a)

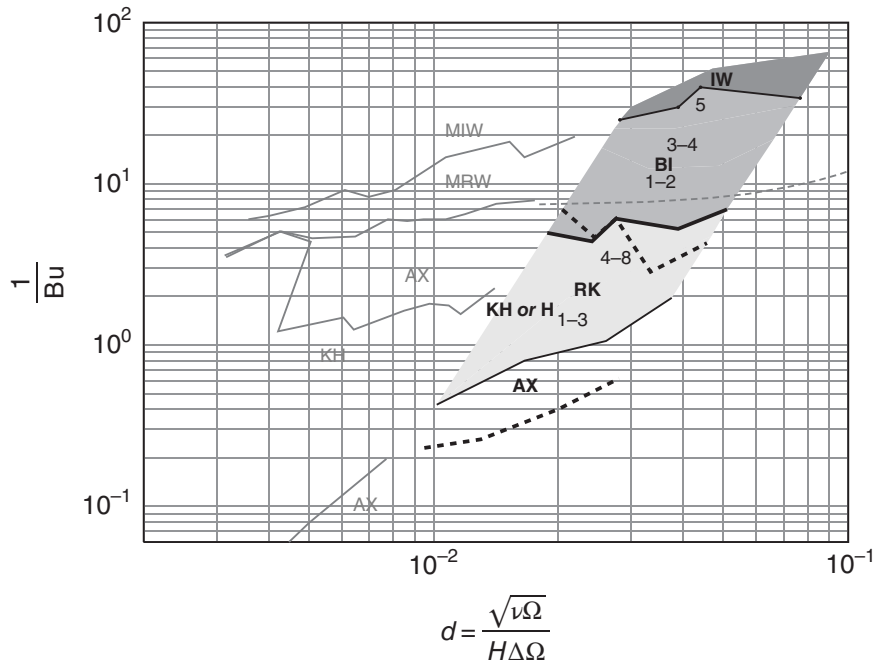

(b)

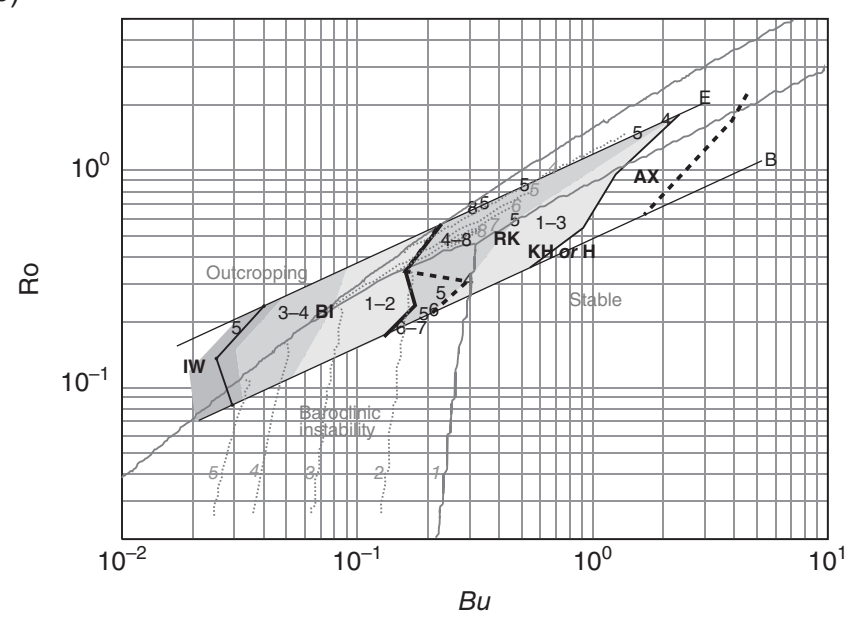

Figure 11.3. Regime diagrams of the different instabilities. (a) in (Bu, d)-space with the gray lines obtained for two-layer immiscible fluids [Williams et al., 2005] separating axisymmetric flow (AX), mixed regular waves (MRWs), and mixed irregular waves (MIWs), and gray shaded areas representing regimes of Kelvin-Helmholtz or Hölmböe instability (KH or H), RK instability, and baroclinic instability (BI) (see Figure 11.2) found for approximately the same density difference $g^{\prime}$ in miscible fluids. The black dashed lines delimit the region of $\mathrm{KH}$ and Hölmböe instability, and the gray dashed line baroclinic instability according to quasi-geostrophic theory [Hart, 1972]; (b) in (Ro, Bu) space with the gray lines representing the theory of Gula et al. [2009a] for inviscid flows. Adapted from Flór et al. [2011].

The required resolution in this case should be estimated from the Batchelor scale for scalar diffusion, defined as $L_{B}=L_{K} \times \mathrm{Sc}^{-1 / 2}[$ Batchelor, 1959; Buch and Dahm, 1996; Rahmani, 2011]. But since singularities due to the relatively high Schmidt number and coarse grid in the present simulations occurred far from the relatively smooth interface, the interfacial dynamics were found to be well captured for a resolution rather estimated with $L_{K}$ (grid step around $1-5$ times $L_{K}$ ).

Given the numerical constraints and purpose of our study on rather small-scale phenomena, we thus found a compromise of a $N_{\theta} \times N_{r} \times N_{z}=1 \times 700 \times 700$ grid with a Schmidt number of 700 and a Reynolds number of 3500 for the study of the secondary circulation. For the $3 \mathrm{D}$ simulation of the flow we choose a grid of $1201 \times 301 \times 257$ with a spatial resolution of $1-5 \mathrm{~mm}$ associated with Schmidt number of 100 and Reynolds number of 10,000 .

Depending on the Reynolds number of the simulation and the associated value of the effective viscosity, the time corresponding to the spin-up took 2-40 dimensionless units.

\subsection{FLOW EVOLUTION OF OBSERVED INSTABILITIES}

Figure 11.3a summarizes the different instability regimes of the interface for a cyclonic forcing and constant density difference as a function of the inverse of the Burger number and dissipation number $d$. The evolution of the instability in parameter space changes from Kelvin-Helmholtz and RK instability to regular baroclinic instability and eventually the irregular change between different baroclinic modes known as the chaotic regime [Flór et al., 2011]. These figures are obtained for increasing background rotation (i.e. for increasing inverse Burger number $1 / \mathrm{Bu}$, and increasing $d$ ) and fixed disk rotation. With respect to results of Williams et al. [2005], Flór et al. [2011] showed RK and Hölmböe instability, both of which will be discussed in more detail in the sections below. When neglecting viscous effects, the full regime diagram reduces to a two-dimensional one as function of Ro and $\mathrm{Bu}$, shown in Figure 11.3b.

Also amplitude vacillation has been observed [e.g., Pedlosky, 1987]. Typically, the oscillation occurs at the limit between RK and baroclinic instability. The flow oscillates in time between the axisymmetric state and the unstable baroclinic state. These periodic changes in wave amplitude are related to the transfer of energy between the wavefield and the zonal flow and have been observed before in two-layer immiscible fluid flows [Hart, 1976, 1979; Read et al., 1992; Früh and Read, 1997] but for smaller $\mathrm{Bu}$ numbers, i.e, in the baroclinically unstable regime. In Figure 11.4a the very contrasted crests and troughs are separated by approximately 35 table rotation periods; the oscillation in mode 2 obtained from these data is represented in Figure 11.4b. Even though there is some noise and the amplitude of the mode gradually decreases, 
(a)

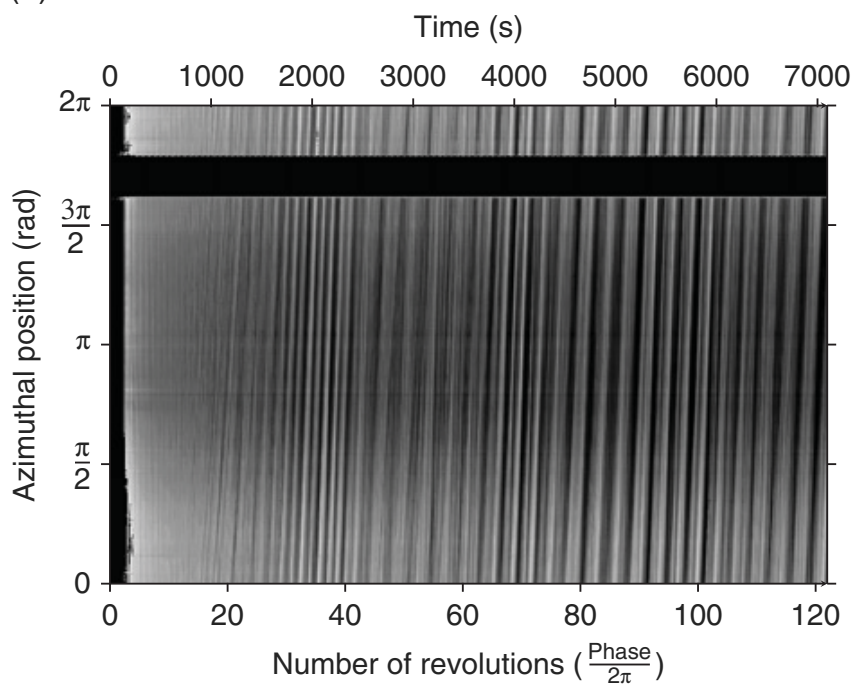

(b)

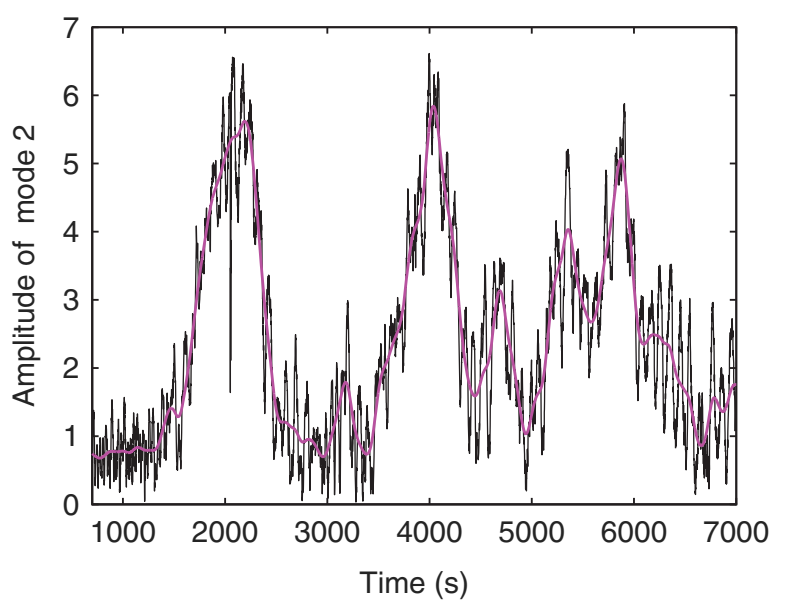

Figure 11.4. Amplitude vacillation of the baroclinic mode 2 shown in a Hovmöller diagram (a) with maxima at approximately 35,70 , and 100 rotation periods ( $x$ axis) and $(b)$ the time evolution of the amplitude of the spatial Fourier mode 2. Experimental parameters: $d=0.023,1 / \mathrm{Bu}=F=3.27$.

this period can clearly be seen. This variation is most likely due to the gradual variation in stratification after more than 100 rotation periods. This confirms the occurrence of amplitude vacillation in two-layer miscible fluids with a smooth interface.

\subsubsection{Rossby-Kelvin Instability}

In order to further investigate the properties of the RK instability, a more specific simulation has been carried out for parameters in the area where both RK instability and Hölmböe instability are expected (region RK in Figure 11.3 with $\mathrm{Bu}=0.62$, Ro $=0.67$ ). For this simulation, the grid resolution is chosen such that small-scale perturbations can be resolved. The Reynolds number and the Schmidt number are 10,000 and 100, respectively, compared to, respectively, 50,000 and 700 in the laboratory experiments (see Figure 11.5).

Figure 11.5 (right side) shows the top views of the interface height and the flow field represented by the stream function of each layer after having subtracted the mean velocity field. The structure in each layer appears clearly different. In the lower layer, linear stability theory predicts the flow to be separated into 12 segments (Figure 11.5b, left). After scrutinizing the image of Figure 11.5b (right), it is indeed possible to identify these 12 segments. Some of the separation lines do not extend to the inner cylinder but merge into a single line. This structure propagates along the outer boundary and can be identified as a Kelvin wave. In the upper layer, the structure of a Rossby wave is well reproduced with the presence of cyclones and anticyclones, and indeed 12 (or 13) cyclonic vortices can be counted. However, due to the interaction between modes, the structure appears not as regular as the linear prediction. A square lower mode structure can be observed on top of the Rossby mode that resonates with the Kelvin mode. Indeed, the temporal evolution of the mode amplitudes reveals that beyond the predominant modes 6 and 5 , lower modes ( 2 and 3 ) are competing, thus confirming a nonlinear interaction between modes (see Figure 11.6). This may explain the observation of much higher growth rates than the growth rates predicted by the linear theory [Gula et al., 2009a]. The general structure is nevertheless in good agreement with the RK instability (Figure 11.5 left side) (Gula, personal communication; see also Gula et al. [2009b]) and provides evidence of the resonance of a Rossby wave in the upper layer and a Kelvin wave propagating along the outer boundary in the lower layer, giving rise to the RK instability.

According to Gula et al. [2009a], the growth rate of the RK instability decreases when the density interface thickness increases. In almost all studies, interfaces of a negligible thickness are considered. Thick interfaces allow for critical layers with a much more complex dynamics. By contrast, the baroclinic instability does not seem to depend on the interface conditions and is not modified by the interface thickness. Nevertheless, Figures 11.3 and 11.6 show that the RK instability appears for higher Burger numbers than the threshold value for baroclinic instability. To further investigate the effect of the interface conditions on the growth rate of the RK instability, preliminary simulations were conducted for various Schmidt numbers and for parameters that are just below the (ageostrophic) threshold for baroclinic 
(a)

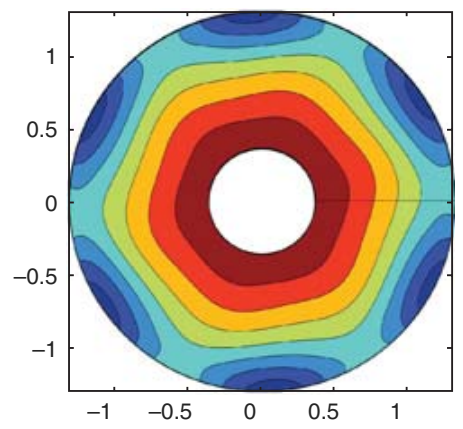

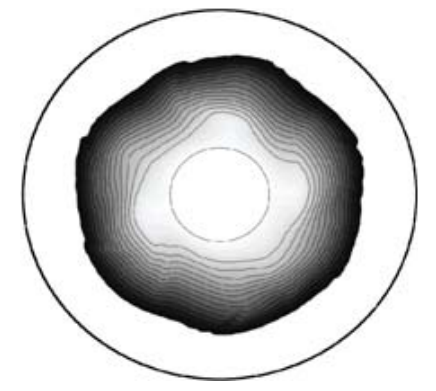

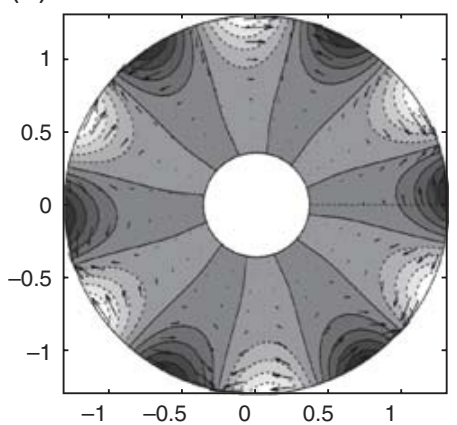

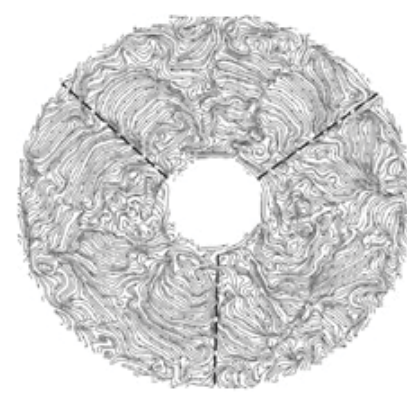

(c)
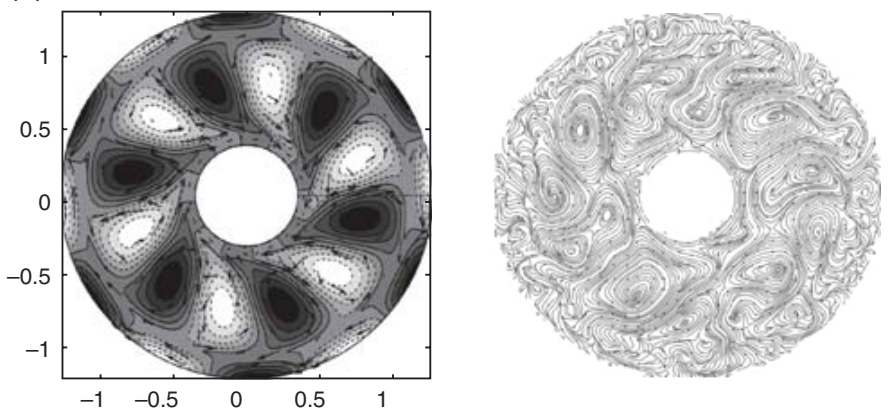

Figure 11.5. Comparison between a high-resolution 3D numerical simulation (right) and linear stability results (left) for the same parameters (Gula, personal communication): (a) interface height field; (b) perturbed velocity field in the lower layer showing a Kelvin wave; (c) perturbed velocity field in the upper layer showing a Rossby wave. The linear stability results also show the pressure field in shading. Numerical parameters: $\mathrm{Bu}=0.62, \mathrm{Ro}=0.67, \Omega=0.077 \mathrm{rad} / \mathrm{s}, \Delta \Omega=0.104 \mathrm{rad} / \mathrm{s}, \mathrm{g}^{\prime}=0.0598 \mathrm{~m}^{2} / \mathrm{s}$, $N_{\theta} \times N_{r} \times N_{z}=1201 \times 301 \times 257$.

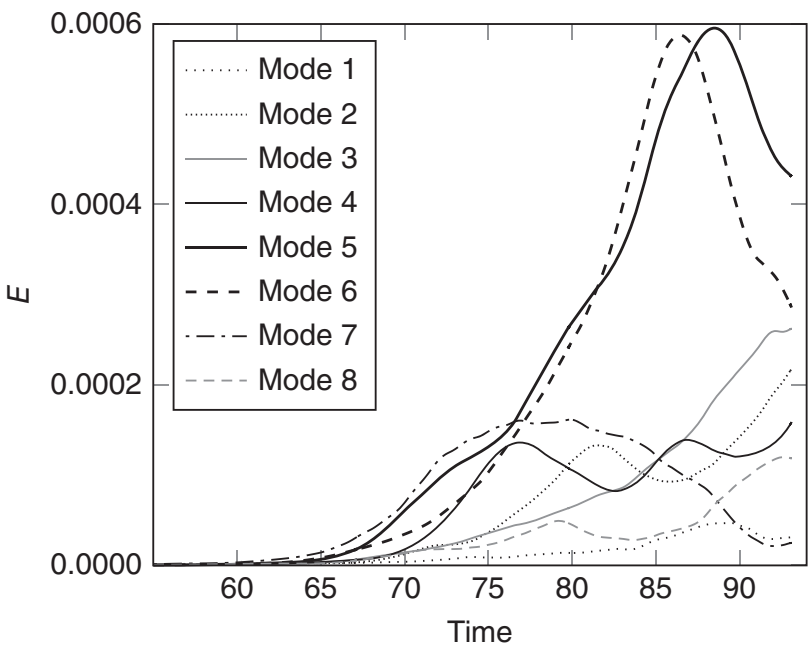

Figure 11.6. Evolution of the energy amplitude of the modes defined by $E(k)=\int_{r} \int_{z} \hat{u} \cdot \hat{u}^{*} d r d z$ with $\hat{u}(r, k, z)$ the Fourier transform of the velocity vector $u(r, \theta, z)$ in the azimuthal direction. Same simulation parameters as in Figure 11.5.

instability (see Figure 11.3). Different Schmidt numbers result in different interface thicknesses, especially for large times. The growth rate of the different amplitudes of the modes show a shift in growth rate with increasing Schmidt number. For low Schmidt numbers, the first growing RK modes are 5 and 6 , whereas a baroclinic mode 2 started to grow at a later instant with a much higher growth rate. The type of instability was identified from the presence of a Rossby or a Kelvin wave in the top or bottom layer, respectively. With increasing Schmidt numbers, the mode number of both instabilities increased, and RK modes 7 and 8 were observed, followed by a faster growing baroclinic mode 4 . These RK modes could well incline locally the interface by an increase in the local shear and thus decrease locally the Burger number. In this manner, the presence of RK modes could act as a finite amplitude perturbation and initiate the baroclinic instability for lower Bu numbers.

\subsubsection{Interface Conditions and Ekman Circulation}

To leading order, the flow generated by the rotating disk at the surface is horizontal. In the vertical, a second-order vertical Ekman circulation is generated by the fluid expelled at the boundary of the rotating disk. In the case of immiscible fluids, this circulation is associated with thin Ekman layers at the internal interface [e.g., van Heijst, 1984]. But when a tanh-like stratification profile is present, such as between two miscible fluids 
(a)

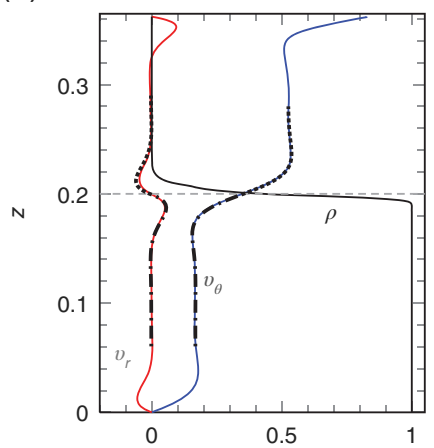

(c)

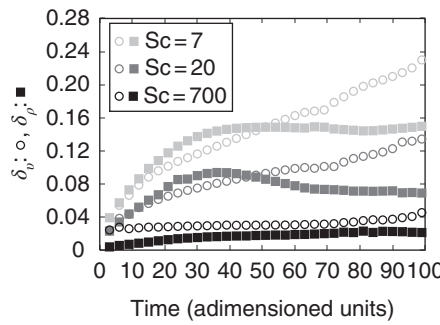

(e)

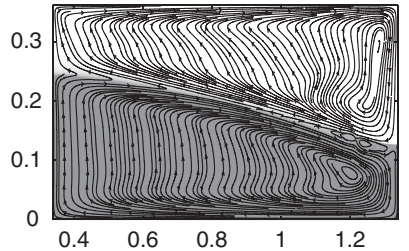

(b)

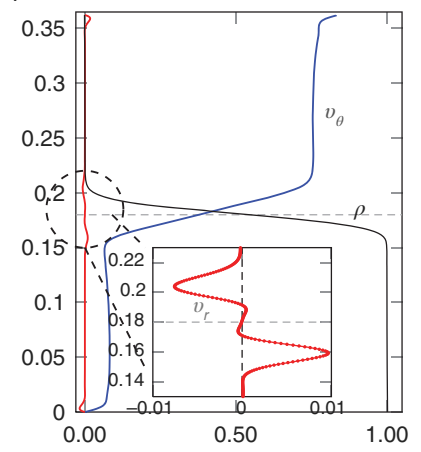

(d)

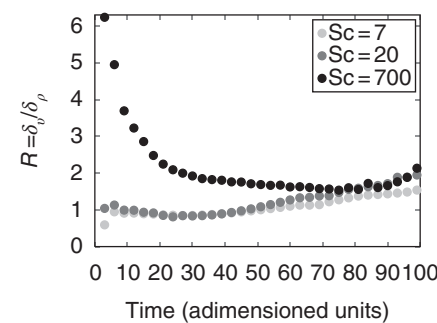

(f)

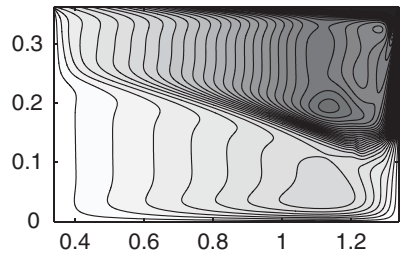

Figure 11.7. Properties of the Ekman layer with azimuthal and radial velocity and density profiles at mid radial position in the annulus after 18 rotation periods for (a) Ro $=0.4861$ and $\operatorname{Re}=3500$ and $(b) \operatorname{Ro}=0.0046$ and $\operatorname{Re}=547.6$. The dotdashed lines represent Ekman layers for a virtual boundary at the interface (the position of the interface is indicated in dashed grey). (c) Interface thicknesses $\delta_{V}(\circ)$ and $\delta_{\rho}(\bullet)$ nondimensionalized by $L$ for Schmidt numbers 7,20 , and 700 and (d) their ratio $R=\delta_{V} / \delta_{\rho}$. In (e) and (f) are shown, respectively, the streamlines and vortex lines along a $r, z$ cross section. The contours of $r v_{\theta}$ are in the range $[-1,1]$ from white to black and background shading for density. Numerical parameters for (a), (e), and (f): $N_{\theta} \times N_{r} \times N_{z}=1 \times 700 \times 700$ and $g^{\prime}=0.06, \mathrm{Bu}=0.3147$, $\mathrm{Sc}=700$.

separated by a diffuse interface, the existence of such internal Ekman layers is not obvious. For the study of the instantaneous density and velocity profiles, we consider this problem numerically. Since the interface has no discontinuities and only axisymmetric stable flows are considered, the numerical modeling is adequate to capture the physics of the flow.

Figure 11.7a shows the profiles of azimuthal velocity, radial velocity, and density of a simulation with high Reynolds number and $\mathrm{Sc}=700$ for a resolution of $1 \times$

$700 \times 700$ supposing axisymmetric flow. Axisymmetric flows still allow for Ekman circulation. The Reynolds number is defined as $R_{e}=\Delta \Omega L^{2} / v$ and is approximately 3500. At $t=0$ the interface is sharp in density and shear. After 18 rotation periods, there is a uniform azimuthal flow in the interior and an Ekman boundary near the interface, as can be deduced from the relative strength of the radial velocity and a variation in azimuthal velocity corresponding to an Ekman spiral. The dot-dashed lines in Figure 11.7a are the velocity profiles as obtained for a classical Ekman layer near a no-slip and zero vertical velocity boundary at the interface, such as for a virtual wall. The perfect agreement between the analytical solution and the numerical result confirms that indeed there are internal Ekman layers above and below the interface. The streamlines and vorticity lines in Figures $11.7 \mathrm{e}$ and $11.7 \mathrm{f}$ show the Ekman layers at each side of the inclined interface in space. In this simulation Ro is not very small (i.e. $\approx 0.48$ ) allowing for some vertical motion and an inclination of the interface (see Figures 11.7e,f).

With increasing interface thickness, the radial velocity and therewith the Ekman pumping reduce in strength such that the flow tends to a unidirectional shear flow, for very thick interfaces. Especially for small Schmidt numbers, diffusion eventually weakens the density gradient so that the stratification and consequently the Burger number decrease. For such small Schmidt numbers the interface is a transient. It inclines more and more with its diffusion, until eventually the fluid is mixed and shear layers are represented by Taylor columns. The interaction of the front with top and bottom solid boundaries and the consequent transport of interfacial fluid via the horizontal and vertical boundary layers enhance the mixing. The evolution is then determined by the limited geometry of the annulus. To consider only the interface dynamics, the simulations are evolved for the time the density transport via the Ekman and Stewartson layers does not significantly affect the density in the bulk of the layers.

Figures $11.7 \mathrm{c}, \mathrm{d}$ show the interface thickness in density and shear (Figure 11.7c) and the interface thickness ratio $R=\delta_{\nu} / \delta_{\kappa}$ as a function of time for different Schmidt numbers (Figure 11.7d). These thicknesses are obtained from fits with a tanh profile. For all $\mathrm{Sc}>1$, the initially very thin density interface increases more or less rapidly in thickness, thus reducing the value of $R$, and eventually reaches a value between 1 and 2. Due to entrainment, the density interface thickness stops growing while the shear thickness continuously increases. The interface thickness ratio $R$ increases to values close to or higher than 2 if we follow the tendency of Figure 11.7d. This evolution shows that the diffusion of vorticity and density across an interface in a rotating fluid is not a linear diffusion problem but is governed rather by entrainment, leading to an eventual larger shear thickness than density thickness. 


\subsubsection{Small Interfacial Waves}

Small-scale waves are observed in the different stages of the experiments reported in various papers on fronts in differentially rotating fluids [Lovegrove et al., 2000; Williams et al., 2005; Flór et al., 2011] (see Figure 11.3). The nature of these waves has been a major question and has been debated in particular as a response to the paper of Williams et al. [2005]. The different candidates that have been considered are adjustment waves, spontaneous emitted inertia-gravity waves [McIntyre, 2009], waves due to Kelvin-Helmholtz or Hölmböe instability, and more recently also the instabilities of the boundary layer at the inner cylinder [Jacoby et al., 2011] and the interfacial Ekman boundary layer [Jacoby, 2012]. Here we briefly review each of these wave types and subsequently focus on experiments in an annulus with a tanh density profile.

Adjustment waves occur when an initially imbalanced flow, during its transient process of adjustment toward a newly balanced geostrophic flow, radiates inertia-gravity waves [Reznik et al., 2001]. Initially equilibrated flows may also radiate inertia-gravity waves, even though the flow is well described by the quasi-geostrophic equations. The emission of these ageostrophic waves are due to a "spontaneous imbalance," a process that may refer to different nonlinear mechanisms. First, these waves can indeed be emitted via a mechanism that is similar to the generation of noise in turbulent flow as described by Lighthill's theory for acoustics. Their radiation have been worked out for the shallow-water equations by Ford [1994]. Another mechanism of wave radiation that has been suggested [Vanneste and Yavneh, 2004] is due to advection: It induces a coupling between equilibrated motion and inertia-gravity waves by a Doppler shift effect.

When gravity waves have a smaller length scale than the balanced motion, the previous mechanisms cannot work since they describe waves with wavelengths of the same order as the large-scale balanced motion. The presence of waves in idealized simulations of baroclinic life cycles in continuously stratified flows [O'Sullivan and Dunkerton, 1995] is due not to the spontaneous emission of inertiagravity waves mentioned above but to the nonlinear development of the baroclinic instability and the particular environment of the flow with strong horizontal deformation and large vertical shear [Plougonven and Snyder, 2005]. In particular, the vertical shear influences the propagation of wave packets. Long waves may either break or be absorbed. This wave capture phenomenon is relevant in shear flows and local wave sources such as, for instance, the radiation by a dipolar vortex in a continuously stratified fluid [Snyder et al., 2007]. The amplitude of these waves is still small compared to the waves due to shear instabilities.

In order to know whether the observed waves are due to shear, we have to consider the Richardson number.
Since the critical (local) Richardson number depends on the interface thickness in shear, $\delta_{v}$, and density, $\delta_{\rho}$, diffusive effects are expected to play an important role for the wavelength and growth of these instabilities. When the interface ratio $R=\delta_{v} / \delta_{\rho}$ exceeds a certain threshold, Hölmböe instability instead of Kelvin-Helmholtz instability is possible. Generally, $R>2$ is needed for Hölmböe instability to occur. Diffusion largely affects the interface thicknesses, especially when the Reynolds number is low, and entrainment is not efficient enough to erode the diffused interface to a certain thickness. Stratified shear instabilities satisfy the general Miles criterion [Miles, 1961, 1963]. If we define the gradient Richardson number, $\operatorname{Ri}(z)=N^{2} /(d U / d z)^{2}$, where $N^{2}(z)=-\left(g / \rho_{0}\right)(\partial \rho / \partial z)$ is the Brunt-Väissälä frequency with a reference density $\rho_{0}$, this criterion states stability for $\operatorname{Ri}(z) \geq \frac{1}{4}$ everywhere in the profile and instability for $\operatorname{Ri}(z)<\frac{1}{4}$. Since gradients are difficult to measure, generally the bulk Richardson number $J$ is used for experimental predictions. Following Hölmböe [Hölmböe, 1962; Lawrence et al., 1991; Ortiz et al., 2002; Tedford et al., 2009; Carpenter, 2009], one can write the dimensionless numbers

$$
J \equiv \frac{g \Delta \rho \delta_{v}}{\rho_{0}(\Delta U)^{2}}, \quad R \equiv \frac{\delta_{v}}{\delta_{\rho}}, \quad a \equiv \frac{2 d}{\delta_{v}},
$$

where $\rho_{0}=\left(\rho_{1}+\rho_{2}\right) / 2, \Delta \rho=\rho_{2}-\rho_{1}, \Delta U=U_{2}-U_{1}$, and $a$ is a potential shift of the center of the velocity profile. For an inviscid Boussinesq fluid, the stability of a stratified shear flow is then given by the Taylor-Goldstein equation [e.g., Hazel, 1972; Pouliquen et al., 1994; Alexakis, 2005, 2007, 2009].

For simplicity, it is generally assumed that $\delta_{v} \gg \delta_{\rho}$, allowing for an idealized model with piecewise constant velocity profile and a jump in density (as in Ortiz et al. [2002]) and illustrated in Figure 11.8a. In such a piecewise model, $J$ keeps the same definition, whereas $a=0$ and (a)

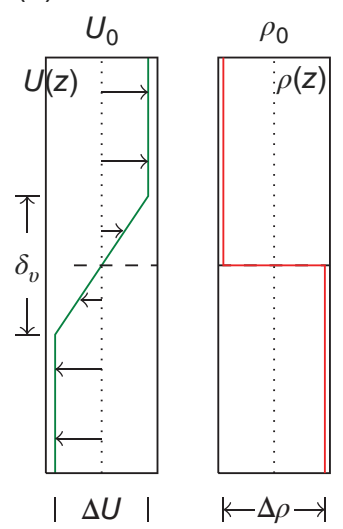

(b)

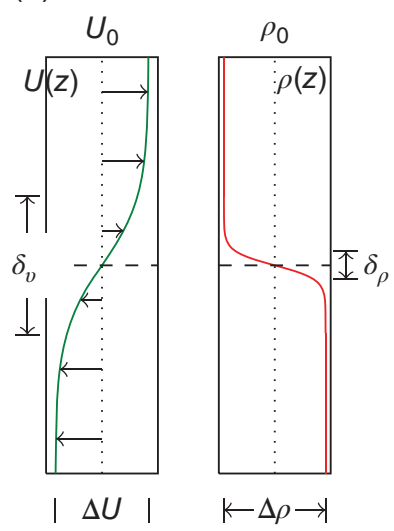

Figure 11.8. Density and velocity profiles in a stratified shear layer: (a) modelization with piecewise profiles; (b) hyperbolic tangent profiles. 
(a)

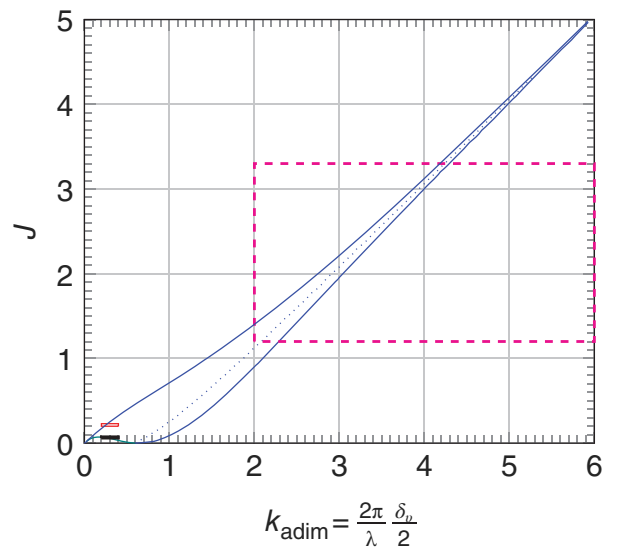

(b)

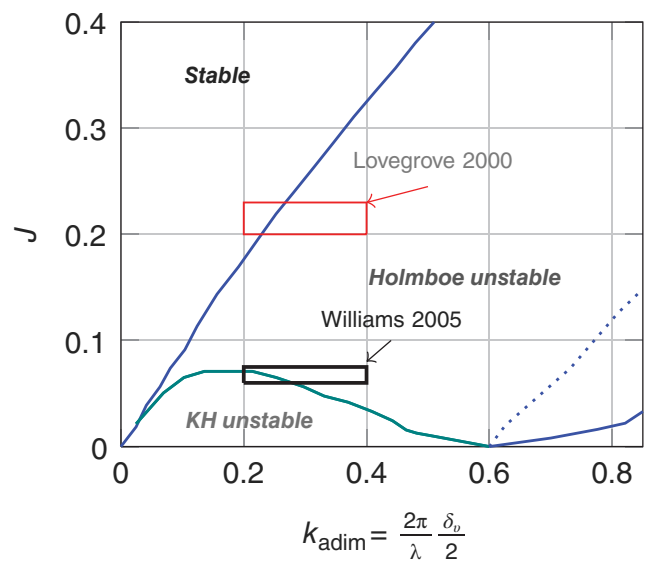

Figure 11.9. The $k-J$ instability diagram for piecewise profiles: (a) experimental areas for interfacial perturbations [Flór et al., 2011] (dashed line) and (b) zoomed in on the regions corresponding to the experiments of Lovegrove et al. [2000] and Williams et al. [2005]. The dimensionless wave number $k=(2 \pi / \lambda)\left(\delta_{V} / 2\right)$ is based on half the shear thickness $\delta_{V} / 2$.

$\delta_{\rho}$ is not relevant. For the small-scale perturbations in the experimental fronts under consideration, the wavenumber and the value of the bulk Richardson number are presented in the $k$-J diagram in Figure 11.9), where we use the dimensionless wave number $k=(2 \pi / \lambda)\left(\delta_{v} / 2\right)$ based on half the shear thickness $\delta_{v} / 2$. Apparently, the experimental data fall in the unstable domain of the Hölmböe instability, especially in the case of experiments with immiscible layers [Lovegrove et al., 2000; Williams et al., 2005] for which the validity of the model is optimal.

However, for a two-layer stratified shear flow with miscible layers, a hyperbolic tangent profile with finite thicknesses in density and in velocity must be taken into account for an appropriate approach of a diffusing density and shear layer (see Figure 11.8b). The variation of the gradient Richardson number varies drastically with $R$ [Alexakis, 2005]. As a consequence, the stability diagram depends also on the thickness ratio (see Figure 11.10). Moreover, the literature on Hölmböe instability has long considered the threshold limit of Hölmböe instability at the value $R=2.2$ (or even 2.4) and the recent results from Alexakis show that the location limit is eventually $R=2$. As illustrated in Figure 11.10 for cases of $R$ just above $R=2$, the unstable Hölmböe domain in the diagram for the global Richardson number $J_{o}=J R$ is very thin and becomes a line at $R=2$. Since $1.84 \leq R \leq 2$, i.e. $R$ is in a part of the regime being located under or equal to 2 , it suggests that (in that part of the regime) these small waves are likely not Hölmböe waves. Indeed, the DNS simulations confirmed the presence of small-scale perturbations superimposed on the RK mode (Figure 11.12) that, because of the values of $R$ and $J$, could not be due to stratified shear instability. Since the value of $R$ increases above 2 for Time $>100$ (see Figures 11.7d and also c,e,f), there will be also a temporal evolution of the stability. (a)

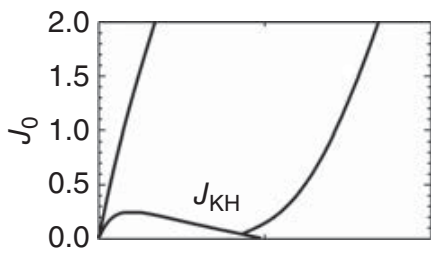

(c)

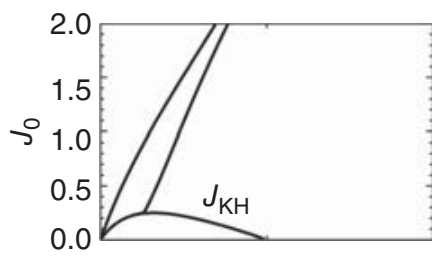

(b)

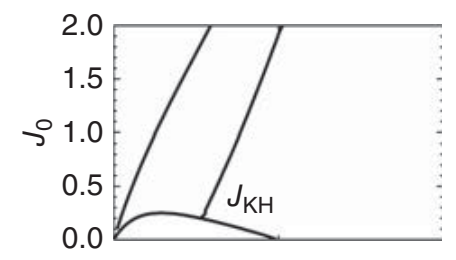

(d)

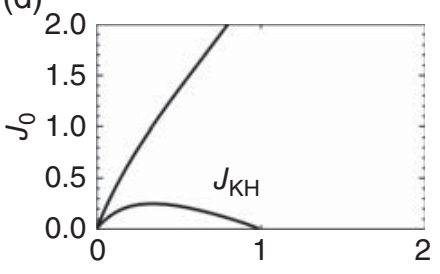

Figure 11.10. Variation of the $k-J_{O}$ diagram $\left(J_{O}=J R\right)$ as a function of the thickness ratio $R$ (from Alexakis [2005]): (a) $R=4$, (b) $R=2.5$, (c) $R=2.2$, (d) $R=2$. In (d) the two stability boundaries $\left(J_{1}\right.$ and $\left.J_{1} S\right)$ have collapsed together on a line and separate the nonsingular gravity wave modes on the left from singular neutral modes on the right.

For $\mathrm{Sc}=700$, initially the ratio $R>2$ so that shear instabilities are likely to occur. Indeed, typical Hölmböe waves (being cusped shaped and with opposite phase direction below and above the interface) have been observed at the beginning of the experiments when the density interface was still relatively sharp (see Figure 11.11).

In the simulations, the ratio $R$ is initially large and subsequently decreases to a critical value close to $R=2$ (see Figure 11.12). Hölmböe instability is therefore possible but should eventually disappear. When the Schmidt number is less than 700 , the asymptotic value tends to a value between 1 and 2, suggesting the possibility of 
(a)

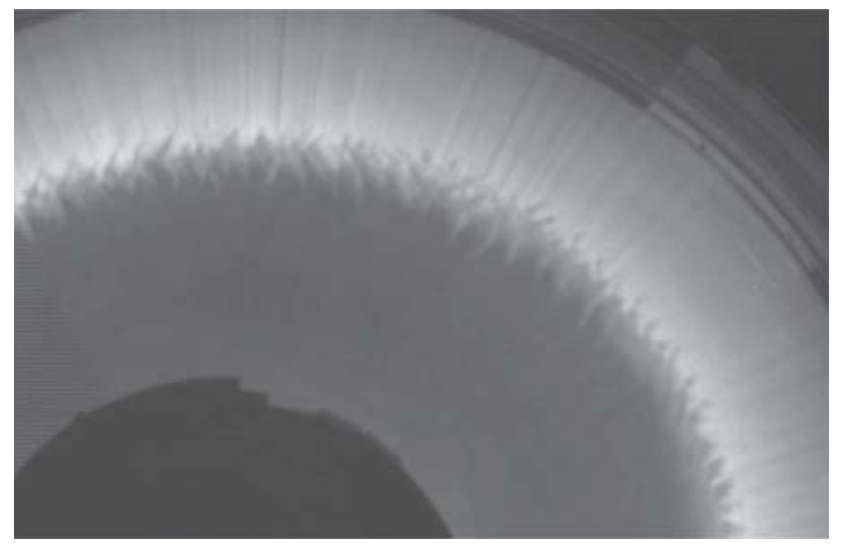

(b)

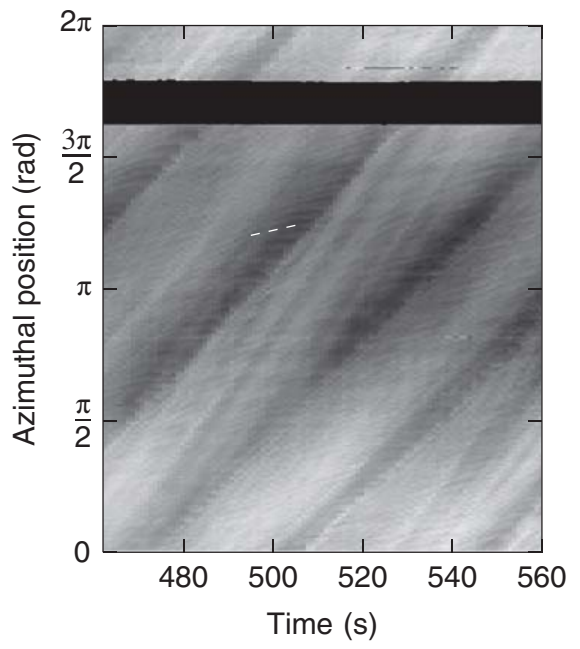

(c)

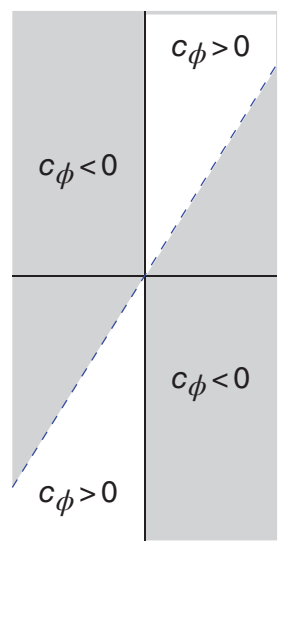

Figure 11.11. Observation of Hölmböe instability, with (a) cusped-shaped wave crests and (b) space-time diagram corresponding to the observation regime. The retrograde propagating Hölmböe waves are characterized by the oblique crests and troughs (indicated by the white dashed line). The diagram in (c) indicates the sign of the phase speed that corresponds to the inclination of the crests with respect to the mean flow (dashed line).

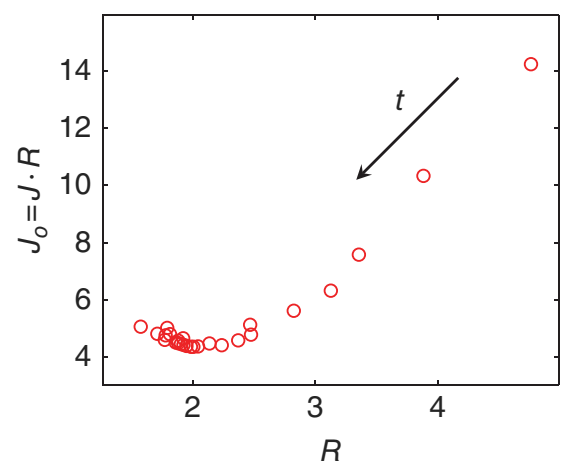

Figure 11.12. Numerical results for the evolution of the values of $R$ and $J_{O}=J R$ as a function of time, with the initial time around $J_{O} \approx 14$ and $R>4$. Note that $R$ decreases below 2 and then increases again in time.

other waves than those due to Hölmböe instability (see Figure 11.7d). The DNS simulations indeed confirmed the presence of small-scale perturbations superimposed on the RK mode (Figure 11.13 and 11.14) that, because of the values of $R$ and $J$, could not be due to stratified shear instability.

The origin of these waves is not understood. Next to the possibility of spontaneous emitted inertia-gravity waves mentioned above, a hypothesis is that the variations in interface slope as induced by the RK instability modes radiate inertia-gravity waves. The ambient presence of the large vertical shear and the presence of horizontal shear are in favor of the wave capture process mentioned above. Since the front is in an ageostrophic state, small-scale adjustment waves are not impossible. Further investigations are necessary to conjecture the type of wave radiation.

\subsection{CONCLUSIONS AND DISCUSSION}

We have focused our attention on experimental and numerical investigations of a baroclinic front in an annular tank. We summarize the results below and end with the consideration of some geophysical flows.

The numerical simulations confirm the presence of a lower layer Kelvin wave and upper layer Rossby wave for parameters in the domain where RK instability was seen experimentally. Preliminary simulations suggest that the change in interface thickness, such as due to different Schmidt numbers, modifies the appearance of instability modes. The growth rates of the higher RK modes increase, and the higher baroclinic modes appear for much lower Burger numbers. A possible mechanism is that the $\mathrm{Bu}$ number locally decreases due to the amplified inclination of the interface at positions where Kelvin waves and Rossby waves interact.

Quasi-geostrophic theory [Hart, 1972] predicts the baroclinic instability at a threshold Burger number of $\mathrm{Bu}<0.12$ (see, e.g., Figure 11.3). Calculations of the ageostrophic baroclinic instability [Gula et al., 2009b] predict the first mode (mode 1 ) at $\mathrm{Bu}<0.3$ and mode 2 at $\mathrm{Bu} \geq 0.2$ being in excellent agreement with the experimental observations, noting that mode 2 is generally the first mode being observed.

Amplitude vacillation of the baroclinic instability seems a general feature in these flows. It appears also for the continuous stratifications here considered, but for larger Burger numbers than the threshold Bu-number value for baroclinic instability. The amplitude of the oscillation decreases in time most likely due to the mixing at the interface and corresponding change in Burger number. 


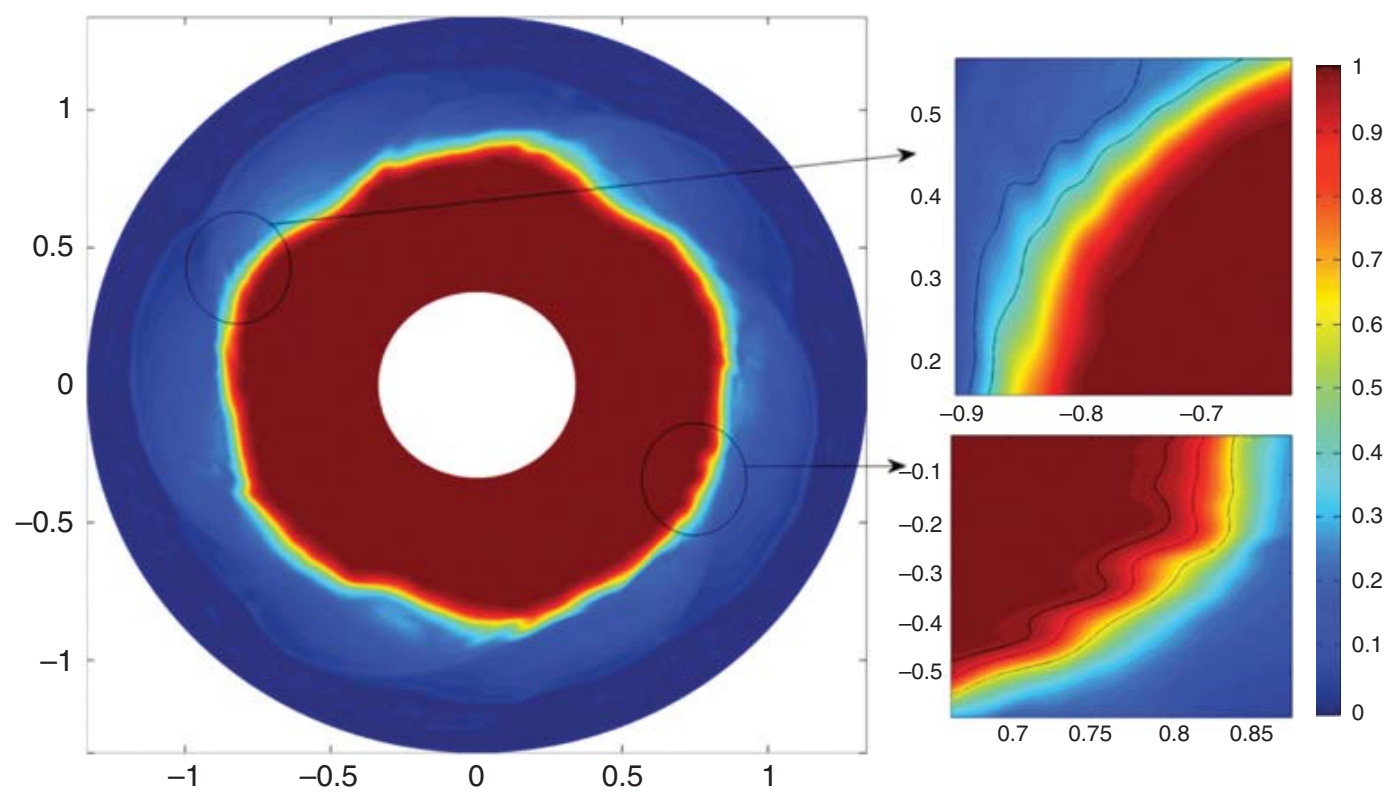

Figure 11.13. Density front for $3 \mathrm{D}$ simulation with $\mathrm{Sc}=100$ for $N_{\theta} \times N_{r} \times N_{z}=1201 \times 301 \times 257, \mathrm{Bu}=0.62$, and Ro $=0.67$. Right: zoom in on the encircled area showing the small-scale perturbations. For color detail, please see color plate section.

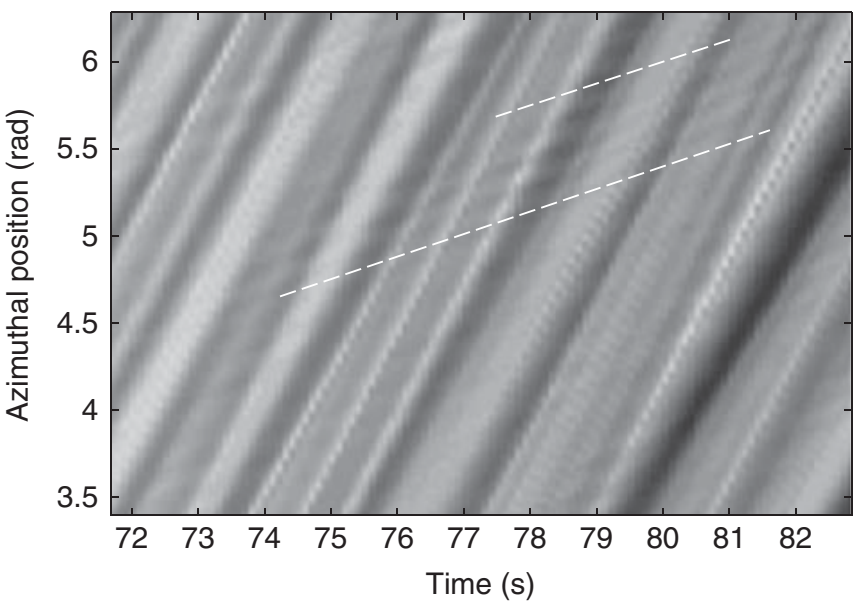

Figure 11.14. Hövmöller diagram, with small-scale perturbations located between the dashed white lines superimposed on the larger wave modes. The small-scale perturbations propagate much slower than the large wave mode. Numerical parameters are as in Figure 11.5.

Internal Ekman layers are considered in relation to interface conditions for different Schmidt numbers. In ageostrophic flows (i.e. for larger Ro numbers) there is an internal Ekman layer when the interface is sharp. But there is a transition to a shear layer (without Ekman pumping) for thicker interfaces and when the Ro number is really small, i.e., $O(0.005)$. In the latter case, the characteristic properties of an Ekman layer (Ekman pumping and vertical circulation) are lost. The effect of strong density diffusion (small Schmidt number) on the Ekman layer is that the Ekman layer disappears and turns into a unidirectional shear layer. For large Schmidt numbers the Ekman layer can be maintained until entrainment modifies the density and velocity distribution.

Small-scale waves have been observed in all experiments. In laboratory experiments, the precise interface conditions are generally not known, so that it is difficult to determine whether Hölmböe, Kevin-Helmhotlz, or other instabilities may occur. Their existence depends on the value of the global Richardson number and the value of the interface thickness ratio, $R$. The knowledge of the Ekman layer and density layer thicknesses in the work of Williams et al. [2005] allows for an accurate estimation of $R$, in contrast to the modeling with miscible fluids in continuously stratified fluids. Varying the Schmidt number modifies the eventual interface thickness. The numerical results show that, only at the start of the experiment when diffusion processes dominate because of the thin interface, the initial value of $R$ may be smaller as well as larger than 2 . Eventually, when entrainment processes dominate, $R$ tends to a minimal value of 2 whereafter it increases again.

A linear diffusion of density and momentum would suggest diffusion rates and related layer thicknesses that are reflected directly by the Schmidt number value. The present results indicate that even for the low values of $\mathrm{Sc}$ values in the atmosphere $(\mathrm{Sc}=1)$ and the ocean $(\mathrm{Sc}=7)$, Hölmböe waves could occur, and the ratio $R$ tends to a value of 2 and increases again afterward (see Figure 11.12) thus allowing for Hölmböe instability.

The RK instability in geophysical flows has so far not been considered. At difference with the baroclinic instability, the Kelvin waves that resonate with the Rossby wave for instability need a boundary along which 
Table 11.2. Estimations of characteristics numbers of large scale oceanic and atmospheric cyclonic flows.

\begin{tabular}{llllllrr}
\hline Geophysical Flows & $N(\mathrm{rad} / \mathrm{s})$ & $\gamma=H /(L / 2)$ & $L(\mathrm{~km})$ & $\mathrm{Bu}$ & Ro & $v_{\text {eddy }}\left(\mathrm{m}^{2} / \mathrm{s}\right)$ & $d$ \\
\hline Antarctic Polar Vortex & $0.01-0.001$ & 0.0023 & 6000 & $0.025-0.9$ & 0.5 & $0.1-10$ & $0.022-0.22$ \\
Cold Core Vortex rings & - & $0.014-0.025$ & $40-70$ & $0.05-0.5$ & $0.1-0.35$ & 0.01 & $0.03-0.05$ \\
Gulf Stream & 0.007 & $0.008-0.012$ & 100 & 0.28 & 0.45 & 0.01 & 0.05 \\
Antarctic Circumpolar Current & 0.002 & $0.1-0.08$ & $35-50$ & $0.64-1.3$ & $0.1-0.2$ & 0.01 & 0.02 \\
Agulhas Current & 0.003 & 0.02 & 93 & 0.19 & $0.02-0.2$ & 0.01 & $0.005-0.02$ \\
\hline
\end{tabular}

Note: For the ocean $v_{\text {eddy }}$ the vertical eddy viscosity is dominated by Ekman layers, i.e. $v=0.01 \mathrm{~m}^{2} / \mathrm{s}$ [Cushman-Roisin, 1994]. For the case of circular currents, the radius or otherwise the half-width current, i.e. $L / 2$ is taken for Ro and Bu number. The estimations for cold-core vortex rings are from Olson [1991] with for the maximum velocity a mean value of $1 \mathrm{~m} / \mathrm{s}$. Antarctic Circumpolar Current values are according to Gille [1994], who measured current widths of 35-50 km driven by surface winds, for which a wavelength of $150 \mathrm{~km}$ was found. The Rossby number is calculated from $U /(f L)$, taking $U=20-40 \mathrm{~cm} / \mathrm{s}$, and for $d$ mean values are used. The values for the Agulhas current are estimated from Webb [1999] and Beal et al. [2006].

they propagate. Therefore most evident applications are oceanic, e.g. coastal fronts. To our knowledge, the possible existence of the RK instability in geophysical flows has not been considered yet. Below we further discuss some geophysical flows and their position in the $\mathrm{Bu}-d$ diagram presented in Figure 11.3.

To compare present results on frontal stability with fronts in nature, estimations of the nondimensional parameter values of some geophysical flows are presented in Table 11.2. The eddy viscosity is related to the friction in the Ekman boundary layer. Further it should be noted that the comparison with oceanic examples concern mainly outcropping flows (since they are visible whereas internal interfaces are not so often observed), in contrast to the present experimental results for internal interfaces. Supposing that the friction at the free surface is not so large to have an important effect on the instability, we compare these flows with the results presented in the diagram of Figure 11.3. The modeled parameter regime set by Ro, Bu numbers and the dissipation number $d$ shown in Figures 11.3a and 11.3b almost covers entirely the parameter space of the real flows represented in Table 11.2. Further, the comparison in Figure 11.3 shows that we may well expect the occurrence of the RK instability in oceanic currents. Wavelengths are about 1 to a $1 / 3$ of the current width, which corresponds to the size of the lenses observed in the ocean near the Gulf Stream, suggesting that the RK instability is a possible candidate that deserves further attention.

Acknowledgments. The authors acknowledge Jonathan Gula for providing the figures for linear stability in Figure 11.5, the contract CIBLE of the French region Rhone Alpes for financing this project, and the calculation centers IDRIS and CINES for the simulation time on their machines. Further, the authors acknowledge two anonymous referees for their corrections to the manuscript.

\section{REFERENCES}

Alexakis, A. (2005), On Holmboe's instability for smooth shear and density profiles, Phys. Fluids, 17, 084,103.

Alexakis, A. (2007), Marginally unstable Holmboe modes, Phys. Fluids, 19, 054,105.

Alexakis, A. (2009), Stratified shear flow instabilities at large Richardson numbers. Phys. Fluids, 21, 054,108.

Appleby, J. (1982), Comparative theoretical and experiment studies of baroclinic waves in a two-layer system, Ph.D. thesis, Leeds University, United Kingdom.

Batchelor, G. K (1959), Small-scale variation of convected quantities like temperature in turbulent fluid. Part 1. General discussion and the case of small conductivity, J. Fluid Mech., 5, 113-133.

Beal, L.M., T.K Chereskin, Y.D Lenn, and S. Elipot (2006). The sources and mixing characteristics of the Agulhas current, J. Phys. Oceanogr., 36, 2060-2074.

Bouruet-Aubertot, P., and P. F Linden (2002), The influence of the coast on the dynamics of upwelling fronts: Part i. Laboratory Experiments, Dyn. Atmos. Oceans, 36(1-3), 153-173.

Bradford, J., A.S. Berman and T.S. Lundgren (1981), Nongeostrophic baroclinic instability in a two-fluid layer rotating system, J. Atmos. Sci., 38, 1376-1389.

Buch, Jr., K. A., and W. J. A. Dahm (1996), Experimental study of the fine-scale structure of conserved scalar mixing in turbulent shear flows. Part 1. Sc >>1, J. Fluid Mech., 317, 21-71.

Carpenter, J. (2009), Unstable waves on a sheared density interface, Ph.D. thesis, University of British Columbia, Vancouver.

Carrigan, C.R. (1978), Instability of a two-layer baroclinic flow in a channel, Tellus, 30, 468-471.

Cenedese, C., and P. F. Linden (2002), Stability of a buoyancydriven coastal current at the shelf break, J. Fluid Mech., 452, 97-121.

Chia, F., R. W. Griffiths, and P. F. Linden (1982), Laboratory experiments on fronts Part II: The formation of cyclonic eddies at upwelling fronts. Geophys. Astrophys. Fluid Dyn., 19, 189-206. 
Cushman-Roisin, B. (1986), Frontal geostrophic dynamics, J. Phys. Oceanogra., 16, 132-143.

Cushman-Roisin, B. (1994), Introduction to Geophysical Fluid Dynamics, Prentice-Hall, Englewood cliffs, NJ.

Eccles, F.J.R., P.L. Read, A.A. Castrejón-Pita, and T.W.N. Haine (2009), Synchronization of modulated traveling baroclinic waves in a periodically forced, rotating fluid annulus, Phys. Rev. E, 79, 1:015202.

Faller, A. J. (1963), An experimental study of the instability of the laminar Ekman boundary layer, J. Fluid Mech., 15, $560-576$.

Flór, J., H. Scolan, and J. Gula (2011). Frontal instabilities and waves in a differentially rotating fluid, J. Fluid Mech., 685, 532-542.

Ford, R. (1994), Gravity wave radiation from vortex trains in rotating shallow water, J. Fluid Mech., 281, 81-118.

Fowlis W. W., and R. Hide (1965), Thermal convection in a rotating annulus of liquid: Effect of viscosity on the transition between axisymmetric and non-axisymmetric flow regimes, J. Atmos. Sci., 22, 541-558.

Fritts, D.C. and M. J. Alexander (2003), Gravity wave dynamics and effects in the middle atmosphere, Rev. Geophys. 41(1), 1003.

Früh, W.G., and P.L. Read (1997), Wave interactions and the transition to chaos of baroclinic waves in a thermally driven rotating annulus, R. Soc. Lond. Philos. Trans. Ser. A, 355, 101-153.

Fultz D., R.R. Long, G.V. Owens, W. Bohan, R. Kaylor and J. Weil (1959), Studies of thermal convection in a rotating cylinder with some implications for large-scale atmospheric motions, Meteorol Monogr. 4(21), 1-104.

Gille. S. T. (1994), Mean sea surface height of the Antarctic Circumpolar Current from GEOSAT data: Methods and application, J. Geophys. Res., 99, 18,255-18,273.

Griffiths, R., and P. Linden (1981), The stability of buoyancydriven coastal currents. Dyn. Atmos. Oceans, 5(4), 281-306.

Gula, J., and V. Zeitlin (2010), Instabilities of buoyancy-driven coastal currents and their nonlinear evolution in the twolayer rotating shallow-water model. Part 1. Passive lower layer, J. Fluid Mech., 659, 69-93.

Gula, J., R. Plougonven, and V. Zeitlin (2009a), Ageostrophic instabilities of fronts in a channel in a stratified rotating fluid, J. Fluid Mech., 627, 485.

Gula, J., V. Zeitlin, and R. Plougonven (2009b), Instabilities of two-layer shallow-water flows with vertical shear in the rotating annulus, J. Fluid Mech., 638, 27.

Gula, J., V. Zeitlin, and F. Bouchut (2010), Instabilities of buoyancy-driven coastal currents and their nonlinear evolution in the two-layer rotating shallow water model. Part 2. Active lower layer, J. Fluid Mech., 665, 209-237.

Hart, J.E. (1972), A laboratory study of baroclinic instability. Geophys. Astrophys. Fluid Dyn., 3, 181-209.

Hart, J.E. (1973), On the behavior of large-amplitude baroclinic waves, J. Atmos. Sci., 30, 1017-1034.

Hart J.E. (1976), The modulation of an unstable baroclinic wave field, J. Atmos. Sci., 33, 1874-1889.

Hart, J. (1979), Finite amplitude baroclinic instability, Ann. Rev. Fluid Mech., 11, 147-172.
Hart, J. (1980), An experimental study of nonlinear baroclinic instability and mode selection in a large basin, Dyn. Atmos. Oceans, 4, 115-135.

Hart, J.E. (1981), Wavenumber selection in nonlinear baroclinic instability, J. Atmos. Sci., 38, 400-408.

Hart, J.E. (1985), A laboratory study of baroclinic chaos on the f-plane, Tellus Ser. A, 37, 286.

Hart, J.E., and S. Kittelman (1986), A method for measuring interfacial wave fields in the laboratory, Geophys. Astrophys. Fluid Dyn., 36(2), 179-185.

Hayashi, Y., and W.R. Young (1987), Stable and unstable shear modes on rotating parallel flows in shallow water, J. Fluid Mech., 184, 477-504.

Hazel, P. (1972), Numerical studies of the stability of inviscid stratified shear flows, J. Fluid Mech., 51, 39-61.

Hide, R. (1953), Some experiments on thermal convection in a rotating liquid, Q. J. R. Meteor. Soc., 79(339), 161-161.

Hide, R. (1958), An experimental study of thermal convection in a rotating liquid, $R$. Soc. Lond. Philos. Trans. Ser. A, 250, $441-478$

Hide, R. (2011) Regimes of sloping thermal convection in a rotating liquid "annulus," Geophys. Astrophys. Fluid Dyn., 105, $11-127$.

Hölmböe, J. (1962), On the behavior of symmetric waves in stratified shear layers. Geofys. Publ., 24, 67-112.

Howard, L.N. (1961), Note on a paper of John W. Miles, J. Fluid Mech., 10, 509-512.

Jacoby, T.N.L. (2012), Inertia-gravity wave generation by boundary layer instabilities, Ph.D. thesis, University of Oxford, Oxford.

Jacoby, T. N. L., P. L. Read, P. D. Williams, and R. M. B. Young, (2011), Generation of inertia?gravity waves in the rotating thermal annulus by a localised boundary layer instability, Geoph. Astr. Fluid Dyn., 105(2-3): 161-181.

King, J.C. (1979), An experimental study of baroclinic wave interactions in a two-layer system, Geophys. Astrophys. Fluid Dyn., 13, 153-167.

Lawrence, G.A., F.K. Browand, and L.G. Redekopp (1991), The stability of a sheared density interface, Phy. Fluids, 3, 2360-2370.

Lovegrove, A.F., P.L. Read, and C.J. Richards (1999), Generation of inertia-gravity waves by a time-dependent baroclinic wave in the laboratory, Phys. Chem. Earth B, 24, 455-460.

Lovegrove, A.F., P.L. Read, and C.J. Richards (2000), Generation of inertia-gravity waves in a baroclinically unstable fluid, Q. J. R. Met. Soc., 126, 3233-3254.

McIntyre, M.,E (2009), Spontaneous imbalance and hybrid vortex-gravity structures, J. Atmos. Sci., 66, 1315-1325.

Miles, J.W. (1961), On the stability of heterogeneous shear flows, J. Fluid Mech., 10, 496-508.

Miles, J.W. (1963), On the stability of heterogeneous shear flows. Part 2. J. Fluid Mech., 16, 209-227.

Olson, D.B. (1991), Rings in the ocean, Ann. Rev. Earth Planet. Sci., 19, 283-311.

Ortiz, S., J. Chomaz, and T. Loiseleux, (2002), Spatial holmboe instability, Phys. Fluids, 14, 2585-2597.

O'sullivan, D., and T.J. Dunkerton (1995), Generation of inertia-gravity waves in a simulated life cycle of baroclinic instability, J. Atmos. Sci., 52, 3695-3716. 
Pedlosky, J. (1964), The stability of currents in the atmosphere and the ocean: Part I, J. Atmos. Sci., 21, 201-219.

Pedlosky, J. (1970), Finite-amplitude baroclinic waves, J. Atmos. Sci., 27, 15-30.

Pedlosky, J. (1987), Geophysical Fluid Dynamics, 2nd ed., Springer-Verlag, New York, 710 pp.

Phillips, N.A. (1954), Energy transformations and meridional circulations associated with simple baroclinic waves in a twolevel, quasi-geostrophic model. Tellus, 6, 273-286.

Plougonven, R. and C. Snyder (2005), Gravity waves excited by jet: Propagation versus generation, Geophys. Res. Lett., 32, L18802.

Plougonven, R., D.J. Muraki and C. Snyder (2005) A Baroclinic instability that couples balanced motions and gravity waves, J. Atmos. Sci., 62, 1545-1559.

Pouliquen, O., J.M. Chomaz, and P. Huerre (1994), Propagating Holmboe waves at the interface between two immiscible fluids, J. Fluid Mech., 266, 277-302.

Rahmani, M. (2011), Kelvin-Helmholtz instabilities in sheared density stratified flows, Ph.D. thesis, University of British Columbia, Vancouver.

Read, P.L., M.J., Bell, D.W. Johnson, and R.M., Small (1992), Quasi-periodic and chaotic flow regimes in a thermally driven, rotating fluid annulus, J. Fluid Mech., 238, 599-632.

Reznik, G.M., V. Zeitlin, and M. Ben Jelloul (2001), Nonlinear theory of geostrophic adjustment. Part 1. Rotating shallowwater model, J. Fluid Mech., 445, 93-120.

Sakai, S. (1989), Rossby-Kelvin instability: A new type of ageostrophic instability caused by a resonance between Rossby waves and gravity waves, J. Fluid Mech., 202, 149-176.

Scolan, H. (2011), Dynamique et stabilité de fronts: phénomènes agéostrophiques, Ph.D. thesis, Université de Grenoble, France.

Scolan, H., R. Verzicco, and J.B. Flór (2011), On density interfaces in rotating fluids, paper presented at the VII International Symposium on Stratified Flows, Rome, Italy.

Snyder, C., D.J. Muraki, R. Plougonven, and F. Zhang (2007), Inertia gravity waves generated within a dipole vortex, J. Atmos. Sci., 64, 4417.

Swarztrauber, P.N. (1974), A direct method for the discrete solution of separable elliptic equations, SIAM J. Numer. Analy, $11,1136-1150$.

Tatro, P. R., and E. L. Mollo-Christensen (1967), Experiments on Ekman layer instability, J. Fluid Mech., 28, 531-543.
Tedford, E.W., J.R. Carpenter, R. Pawlowicz, R. Pieters, and G.A. Lawrence (2009), Observation and analysis of shear instability in the Fraser River estuary, J. Geophys. Res. (Oceans), 114, C13, C11006.

van Heijst, G.J.F. (1984), Frontal upwelling in a rotating twolayer fluid, Geophys. Astrophys. Fluid Dyn., 29, 139-153.

Vanneste, J., and I. Yavneh (2004), Exponentially small inertia gravity waves and the breakdown of quasigeostrophic balance. J. Atmos. Sci., 61, 211-223.

Verzicco, R., and R. Camussi (1997), Transitional regimes of low-Prandtl thermal convection in a cylindrical cell. Phys. Fluids, 9, 1287-1295.

Verzicco, R. and P. Orlandi (1996), A finite-difference scheme for three-dimensional incompressible flows in cylindrical coordinates, J. Computat. Phys., 123(2), 402-414.

Verzicco, R., F. Lalli, and E. Campana (1997), Dynamics of baroclinic vortices in a rotating, stratified fluid: A numerical study, Phys. Fluids, 9, 419-432.

Webb, D.J. (1999), An analytic model of the Agulhas Current as a Western Boundary current with linearly varying viscosity J. Phys. Oceanogr., 29, 1517-1527.

Williams, P.D., P.L. Read, and T.W.N. Haine (2003),. Spontaneous generation and impact of inertia-gravity waves in a stratified, two-layer shear flow, Geophys. Res. Lett., 30, 24, 240000-1.

Williams, P.D., T.W.N. Haine, and P.L. Read (2004a). Stochastic resonance in a nonlinear model of a rotating, stratified shear flow, with a simple stochastic inertia-gravity wave parameterization. Nonlin. Process. Geophys., 11, 127-135.

Williams, P.D., P.L. Read, and T.W.N. Haine (2004b). A calibrated, non-invasive method for measuring the internal interface height field at high resolution in the rotating, two-layer annulus, Geophys. Astrophys. Fluid Dyn., 98, 453-471.

Williams, P.D., T.W.N. Haine, and P.L. Read (2005), On the generation mechanisms of short-scale unbalanced modes in rotating two-layer flows with vertical shear, J. Fluid Mech, 528:1-22.

Williams, P.D., T.W.N. Haine, and P.L. Read (2008), Inertiagravity waves emitted from balanced flow: Observations, properties, and consequences, J. Atmos. Sci., 65, 3543.

Williams, P.D., P L. Read, and T.W.N. Haine (2010), Testing the limits of quasi-geostrophic theory: Application to observed laboratory flows outside the quasi-geostrophic regime, J. Fluid Mech., 649, 187-203. 